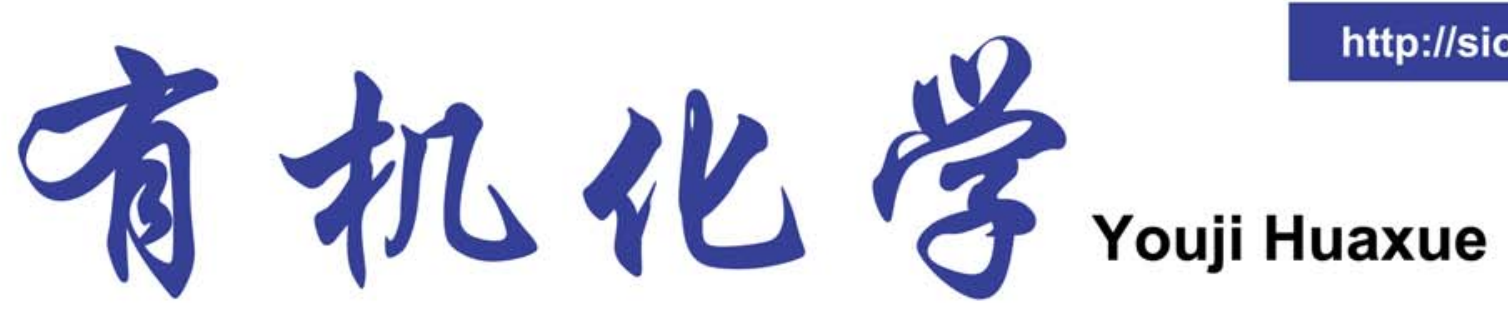 \\ Chinese Journal of Organic Chemistry
}

\section{第33卷 第11期 Vol. 33 No.11 2013}
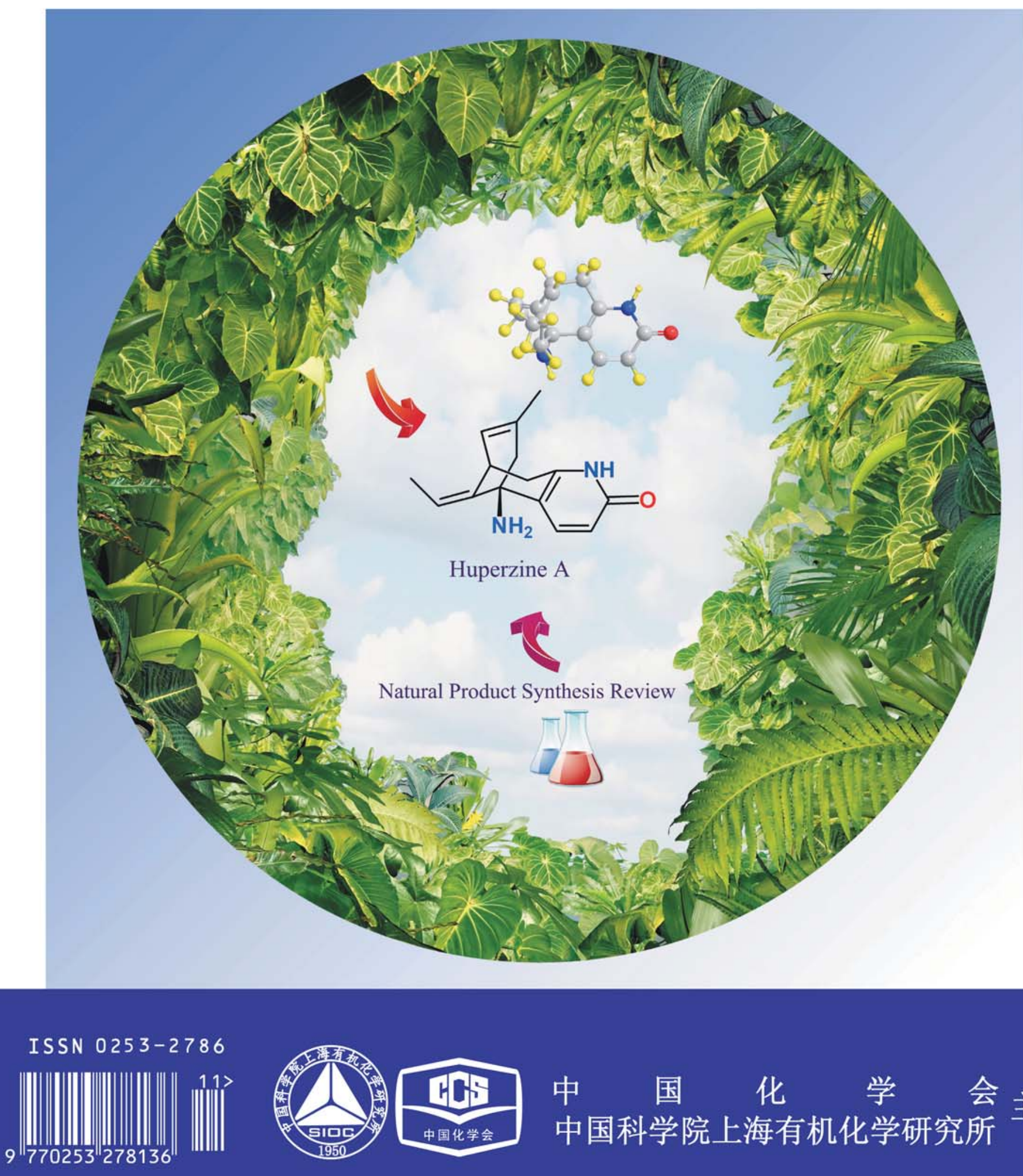

中国 化 学 会 主办
中国科学院上海有机化学研究所 


\section{Born to find out}
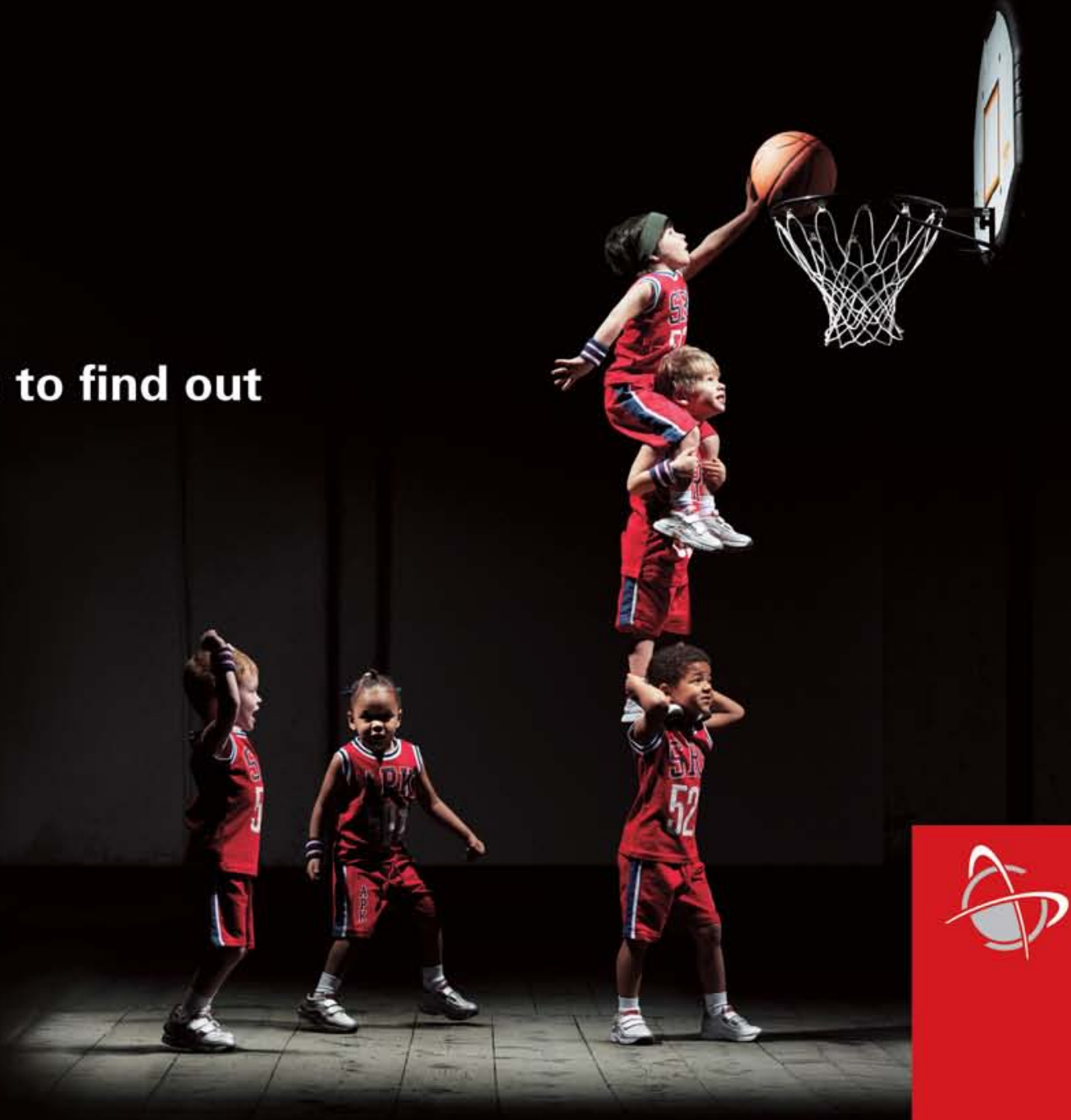

Anton Paar

安东帕 (中国) 有限公司 电话 : 021-6485 5000

传真 : 021-6485 5668

Email: Info.cn@anton-paar.com www.anton-paar.com.cn

\section{安东帕创新分析测试解决方案}

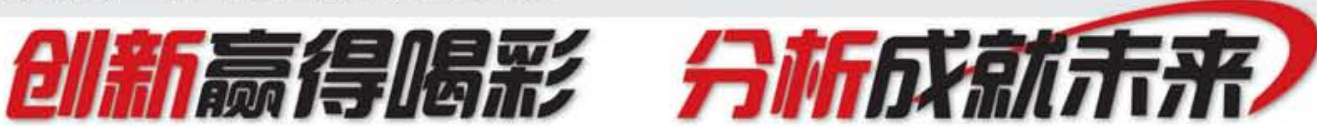

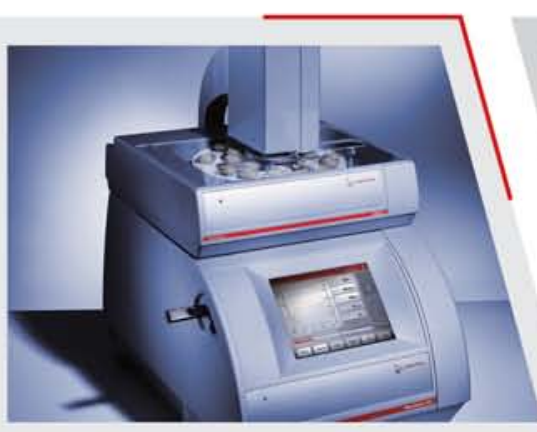

单模微波反应器

Monowave $300+$ MAS 24

一最高微波反应条件: $300^{\circ} \mathrm{C} @ 30 \mathrm{bar}$

一特殊的碳化硅反应管可用于微波机理研究

一实际反应温度和红外温度可同时监控

一叠加式自动进样 - 节省实验室空间

新药研发、金属催化、固相合成及纳米材 料合成等合成反应的方法开发 ( $\mathrm{mg}$ to $\mathrm{g}$ )

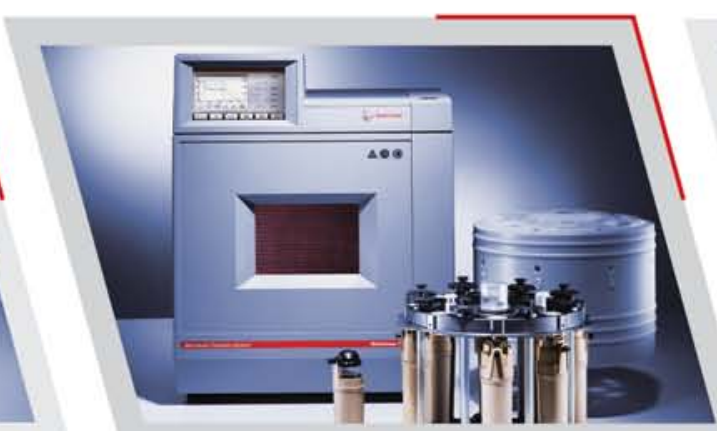

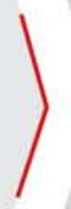

高性能多模微波反应系统

Multiwave PRO

- 可靠的温度和压力传感器 - 最佳反应控制

最高微波反应条件 $\left(300^{\circ} \mathrm{C}\right.$ 和 $\left.80 \mathrm{bar}\right)$ - 拓展了 微波合成的应用范围

一专业的充气装置 一 轻松实现情性气体保护

模块化扩展功能 - 同一主机适用所有类型转子

新药研发、生物医学科学、纳米材料研究、法医鉴 定学等合成的放大反应 ( $\mathrm{g}$ to $\leq 100 \mathrm{~g}$ )

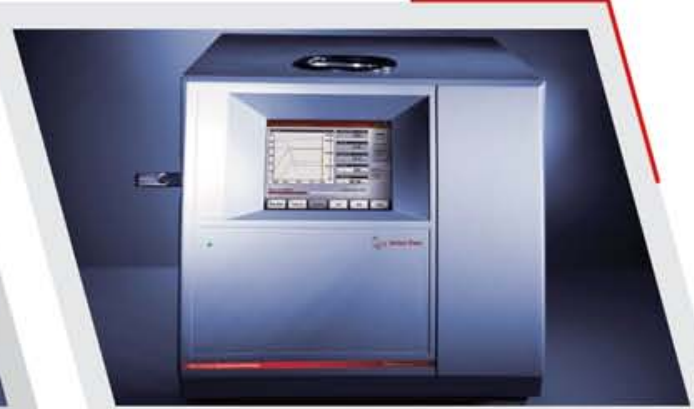

公斤级微波反应器

Masterwave BTR

一最高微波反应条件: $250^{\circ} \mathrm{C} @ 30 \mathrm{bar}$ - 无可比拟的生产力: 公斤级产量

磁力驱动浆式摚拌装置可自动调整搅拌速度 独特冷却安全系统保证公斤级反应安全

新药研发、有机杂环合成、金属催化、固相合成及 纳米材料合成等合成反应的公斤级放大 $(50-300 \mathrm{~g})$ 


\title{
金属催化芳基硼酸与酮不对称 1,2-加成反应研究进展
}

\author{
罗人仕廖建华张 剑* \\ (赣南医学院药学院 赣州 341000)
}

\begin{abstract}
摘要 许多具有生物活性的天然产物及药物均含有手性芳基叔醇结构单元，金属催化芳基硼酸与酮不对称 1,2-加成反 应是合成该类化合物的有效方法. 由于芳基硼酸具有官能团兼容性好、对水及空气稳定、低毒等优点，近来已经成为 化学家研究的热点. 就近年来金属催化芳基硼酸与活泼酮如静红、三氟甲基酮、 $\alpha$-酮酯及 1,2-二酮和不活泼酮的不对称 1,2-加成的研究进展做一详细综述.
\end{abstract}

关键词 金属催化; 芳基硼酸; 酮; 不对称; 1,2-加成; 研究进展

\section{Progress in the Asymmetric Metal-Catalyzed 1,2-Addition Reactions of Arylboronic Acids with Ketones}

\author{
Luo, Renshi Liao, Jianhua Zhang, Jian* \\ (School of Pharmaceutical Sciences, Gannan Medical University, Ganzhou 341000)
}

\begin{abstract}
A number of biologically active compounds and drugs contain the structural unit of chiral aryl-substituted tertiary alcohol. The catalytic asymmetric arylation of ketones is a powerful tool for the efficient construction of such compounds. Arylboron reagents have attracted considerable attention due to their availability, relative insensitivity to air and moisture, high functional group tolerance and generally low toxicity. Recent progress of the catalytic asymmetric arylation of highly activated ketones such as isatins, trifluoromethyl ketones, $\alpha$-ketoesters, 1,2-diketones and unactivated ketones is reviewed.
\end{abstract}

Keywords metal-catalyzed; arylboronic acid; ketone; asymmetric; 1,2-addition; progress

许多具有生物活性的天然产物及药物具有芳基取 代的手性叔醇片段 ${ }^{[1]}$, 因此该类手性化合物越来越引起 化学家的研究兴趣. 随着时代对手性药物的需求与发 展，该类化合物的合成也成为研究的热点 ${ }^{[2]}$. 叔醇化合 物的合成可以使用格氏试剂或者芳基锂试剂对酮进行 亲核加成反应获得, 此外有机锡 ${ }^{[3]}$ 、锌 ${ }^{[4]}$ 、硅 ${ }^{[5]}$ 、钛 ${ }^{[6]}$ 对 酮的加成也是常用的合成方法. 但上述所使用的试剂具 有许多缺点: (1)格氏试剂活性太高及官能团兼容性不 好; (2)锡试剂的高毒性; (3)有机锌试剂、有机硅试剂和 有机钛试剂制备繁琐. 以上的不足使得以往的方法受到 了很大的限制.

1998 年 Miyaura 小组第一次报道使用 $[\mathrm{Rh}(\mathrm{acac})-$ $(\mathrm{CO})_{2}$ ] 与 1,1'-双(二苯基膦)二茂铁(DPPF)催化芳基硼酸 与醛发生 1, 2-加成获得很好的收率 ${ }^{[7]}$. 因芳基硼酸具有 官能团兼容性好、对水以及空气稳定、低毒等优点, 且 使用合适的手性配体可获得高收率及对映选择性的芳
基取代手性醇加成产物. 所以该小组的研究工作自报道 以来引起化学家们的极大关注, 随后许多课题小组对该 领域进行了较为深入的研究 ${ }^{[8 \sim 13]}$. 目前该研究领域已有 较为详细的综述 ${ }^{[14]}$. 在成功研究芳基嗍酸与醛的加成 反应的同时，化学家们也开始研究金属催化芳基嗍酸与 酮的不对称加成反应. 然而与醛相比, 酮的不对称 1,2加成反应的收率与对映选择性均不理想. 究其原因: 一 是酮的反应活性较低; 二是与醛相比酮的羰基两侧取代 基的位阻差异较小使得该类底物的不对称催化难以实 现. 基于上述原因，目前金属催化酮的不对称 1,2-加成 反应的报道极少且成功报道的例子主要是活性较高的 酮，如: 靛红、三氟甲基酮、 $\alpha$-酮酯及 1,2-二酮类化合物 ${ }^{[15]}$ 或者是分子内的芳基硼酸与酮的加成. 令人惊喜的 是最近分子间芳基硼酸与不活泼酮如苯乙酮的不对称 加成也获得了很好的进展. 虽然分子间芳基硼酸与不活 泼酮的对映选择性不尽如人意, 但为其进一步研究提供

\footnotetext{
* E-mail: zhangjiangnmc031@163.com

Received May 27, 2013; revised June 24, 2013; published online July 3, 2013.

Project supported by the Science Foundation of Gannan Medical University (No. YB201014)

赣南医学院项目课题(No. YB201014)资助项目.
} 
了很好的借鉴作用. 本文就近年来报道的金属催化不对 称芳基硼酸与酮的 1,2-加成反应做一详细综述.

\section{1 靛红}

手性吲哚酮是许多天然产物及具有生物活性化合 物的结构单元, 如化合物 I 是一种非常有效的生长激素 促分泌素, 化合物 II 是对 Maxi-K 通道一类有效的激活 剂(图 1).<smiles>CCCCCN1C(=O)[C@@](O)(c2ccccc2Cl)c2c1cc(C(N)=O)cc2C(F)(F)F</smiles><smiles>O=C1Nc2cc(C(F)(F)F)ccc2[C@]1(O)c1cc(Cl)ccc1O</smiles>

图 1 具有生物活性手性 3-着基-3-芳基吲哚酮化合物

Figure 1 Biologically active compounds featuring a 3-aryl-3hydroxy-2-oxindoles motif

由于该类化合物的重要性, 其不对称合成成为非常 有价值的研究工作, 不对称催化芳基硼酸与靛红的芳基 化反应是合成该类化合物最直接有效的方法. 2006 年, Minnaard 等 ${ }^{[16]}$ 报道了 $\left[\left(\mathrm{C}_{2} \mathrm{H}_{4}\right)_{2} \mathrm{Rh}(\mathrm{acac})\right]$ 与手性 N,P 配体 $\mathbf{L 1}$ 催化芳基硼酸与静红的不对称加成反应. 此外, Hayashi 小组 ${ }^{[18]}$ 报道使用手性单膦配体 $(R)-\mathrm{MeO}-\mathrm{mop}$ 与 $\left[\mathrm{RhCl}\left(\mathrm{C}_{2} \mathrm{H}_{4}\right)_{2}\right]_{2}$ 催化芳基硼酸与青定红的不对称加成可获 得更好对映选择性 (图 2). 该部分研究工作在 2009 年 Wiley 出版的 《Modern Arylation Methods》一书中 Carreira 教授 ${ }^{[19]}$ 也进行了较为详细的综述.
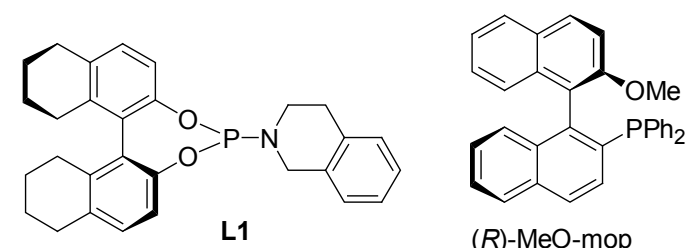

图 2 用于芳基嗍酸与靛红不对称加成的手性配体

Figure 2 Chiral ligands used in the asymmetric reactions

2009 年, Qin 等 ${ }^{[20]}$ 报道使用联苯二溴化合物为原料 合成手性膦亚胺化合物 $(R, S)-\mathbf{L} 2$ 并用于芳基硼酸与靛红 的不对称加成. 作者发现使用 $\mathrm{Rh}\left(\mathrm{CH}_{3} \mathrm{CN}\right)(\mathrm{cod}) \mathrm{BF}_{4}$ 与 $(R, S)-\mathbf{L} 2$ 催化反应只获得消旋的加成产物, 可能的原因 是 $\operatorname{cod}$ 的配位能力强于 $(R, S)-\mathbf{L} 2$, 因此只发生背景, 反应 获得非手性产物. 使用 $\operatorname{Pd}(\mathrm{OAc})_{2}$ 无背景反应发生并可 获得较好的对映选择性, 但反应的活性较低. 作者发现 添加路易斯酸如 $\mathrm{BF}_{3} \mathrm{Et}_{2} \mathrm{O}$ 可活化反应底物提高反应活 性, 遗憾的是收率与选择性均没有明显的提高, 只获得 中等的收率与对映选择性(表 1).

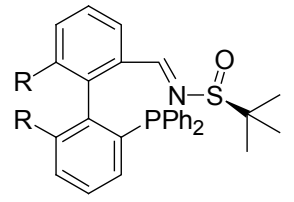

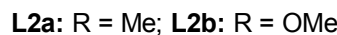

$\mathrm{Pd}(\mathrm{OAc})_{2}(5 \mathrm{~mol} \%)$

$(R, S)$-L2 (10 mol\%)

$\mathrm{BF}_{3} \cdot \mathrm{Et}_{2} \mathrm{O}$ (4 equiv.) THF/r.t./48 h

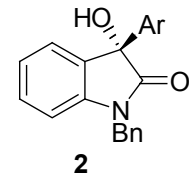

表 1 配体 $\mathbf{L} 2$ 催化的芳基硼酸与 $\mathbf{1}$ 的不对称加成 ${ }^{a}$

Table 1 Asymmetric addition of arylboronic acids to 1 catalyzed by ligands $\mathbf{L} \mathbf{2}$

\begin{tabular}{ccccc}
\hline Entry & Ligand & \multicolumn{1}{c}{$\mathrm{Ar}$} & Yield $^{b} / \% e e^{c} / \%$ \\
\hline 1 & $(R, S)$-L2a & $(-)-\mathbf{2 a}(\mathrm{Ar}=\mathrm{Ph})$ & 63 & 67 \\
2 & $(S, S)$-L2a & $(+)-\mathbf{2 a}(\mathrm{Ar}=\mathrm{Ph})$ & 48 & 62 \\
3 & $(R, S)$-L2b $(-)-\mathbf{2 a}(\mathrm{Ar}=\mathrm{Ph})$ & 40 & 60 \\
4 & $(S, S)$-L2b $(+)-\mathbf{2 a}(\mathrm{Ar}=\mathrm{Ph})$ & 30 & 57 \\
5 & $(R, S)$-L2a $(+)-\mathbf{2 b}\left(\mathrm{Ar}=4-\mathrm{MeOC}_{6} \mathrm{H}_{4}\right)$ & 78 & 38 \\
6 & $(R, S)$-L2a $(-)-\mathbf{c c}\left(\mathrm{Ar}=3-\mathrm{MeOC}_{6} \mathrm{H}_{4}\right)$ & 51 & 73 \\
7 & $(S, S)$-L2a & $(+)-\mathbf{2 d}\left(\mathrm{Ar}=4-\mathrm{FC}_{6} \mathrm{H}_{4}\right)$ & 36 & 65 \\
\hline
\end{tabular}

${ }^{a} 5 \mathrm{~mol} \% \mathrm{M}, 10 \mathrm{~mol} \%(R, S)$-L2a, 0.5 equiv. $t$-BuOK. ${ }^{b}$ Isolated yield. ${ }^{c}$ ee values were determined by HPLC on a Chiralcel OD-H or AD-H column with hexane/2-propanol.

Liao 等 ${ }^{[21]}$ 则报道了更为简单的手性亚砜膦化合物 L3c 与 $\left[\mathrm{RhCl}\left(\mathrm{C}_{2} \mathrm{H}_{4}\right)_{2}\right]_{2}$ 催化芳基硼酸和靛红的加成反应, 以 $\mathrm{MeOH}$ 为溶剂, 以 $5.0 \mathrm{~mol} \%$ DIPEA 为碱, 在 $40{ }^{\circ} \mathrm{C}$ 下 反应 $3 \mathrm{~h}$ 后可获得 $80 \% \sim 99 \%$ 的收率及 $83 \% \sim 95 \%$ ee (Eq. 2).<smiles>[R]c1ccc(S(=O)C(C)(C)C)c(-c2ccccc2)c1[R]</smiles>

L3a: $\mathrm{R}^{1}=\mathrm{H}, \mathrm{R}^{2}=\mathrm{OCH}_{2} \mathrm{OCH}_{3}$ L3b: $\mathrm{R}^{1}=\mathrm{H}, \mathrm{R}^{2}=\mathrm{OCH}_{3}$ L3c: $\mathrm{R}^{1}=\mathrm{R}^{2}=\mathrm{OCH}_{3}$<smiles>[R]c1ccc2c(c1)C(=O)C(=O)N2</smiles>

$\mathrm{R}=\mathrm{H}, \mathrm{Cl}, \mathrm{F}, \mathrm{CH}_{3}$
$\left[\mathrm{Rh}\left(\mathrm{C}_{2} \mathrm{H}_{4}\right)_{2} \mathrm{Cl}\right]_{2}(2.0 \mathrm{~mol} \%)$

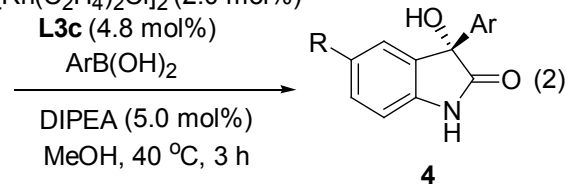

手性联菜酚衍生的齿类亚磷酰胺配体 $(R, R)-\mathrm{Me}-$ BIPAM 是 Yamamoto 研究小组 ${ }^{[2]}$ 发展起来的一类有效 的手性配体，该类配体与 $\mathrm{Ru}$ 在醛、酮酯及乙醛酸的不 对称芳基化反应中表现出很好的催化性能. 最近作者将 其应用到静红的不对称芳基化反应中也可获得了不错 的反应结果 (Eq. 3) (表 3) ${ }^{[23]}$. 
表 2 Rh-催化苯基硼酸与靛红的不对称加成 ${ }^{a}$

Table 2 Rhodium-catalyzed asymmetric addition of arylboronic acids to isatins

\begin{tabular}{cclccc}
\hline Entry & $\mathrm{R}$ & \multicolumn{1}{c}{$\mathrm{Ar}$} & $\mathbf{4}$ & Yield $^{b} / \%$ & $e e^{c} / \%$ \\
\hline 1 & $\mathrm{H}$ & $\mathrm{C}_{6} \mathrm{H}_{5}$ & $\mathbf{4 a}$ & 97 & 85 \\
2 & $\mathrm{Cl}$ & $\mathrm{C}_{6} \mathrm{H}_{5}$ & $\mathbf{4 b}$ & 96 & 91 \\
3 & $\mathrm{~F}$ & $\mathrm{C}_{6} \mathrm{H}_{5}$ & $\mathbf{4 c}$ & 97 & 88 \\
4 & $\mathrm{Me}$ & $\mathrm{C}_{6} \mathrm{H}_{5}$ & $\mathbf{4 d}$ & 94 & 86 \\
5 & $\mathrm{Cl}$ & $2-\mathrm{MeC}_{6} \mathrm{H}_{4}$ & $\mathbf{4 e}$ & 95 & 92 \\
6 & $\mathrm{Cl}$ & $3-\mathrm{MeC}_{6} \mathrm{H}_{4}$ & $\mathbf{4 f}$ & 99 & 91 \\
7 & $\mathrm{Cl}$ & $3-\mathrm{MeOC}_{6} \mathrm{H}_{4}$ & $\mathbf{4 g}$ & 95 & 90 \\
8 & $\mathrm{Cl}$ & $4-\mathrm{MeOC}_{6} \mathrm{H}_{4}$ & $\mathbf{4 h}$ & 96 & 87 \\
9 & $\mathrm{Cl}$ & $4-\mathrm{FC}_{6} \mathrm{H}_{4}$ & $\mathbf{4 i}$ & 98 & 87 \\
10 & $\mathrm{Cl}$ & $4-\mathrm{MeC}_{6} \mathrm{H}_{4}$ & $\mathbf{4 j}$ & 97 & 90 \\
11 & $\mathrm{Cl}$ & $4-t-\mathrm{BuC}_{6} \mathrm{H}_{4}$ & $\mathbf{4 k}$ & 95 & 91 \\
12 & $\mathrm{Cl}$ & $4-\mathrm{BnOC}_{6} \mathrm{H}_{4}$ & $\mathbf{4 l}$ & 96 & 87 \\
13 & $\mathrm{Cl}$ & $2,4-\mathrm{Me}_{2} \mathrm{C}_{6} \mathrm{H}_{3}$ & $\mathbf{4 m}$ & 80 & 83 \\
\hline
\end{tabular}

${ }^{a}$ Reaction conditions: $0.2 \mathrm{mmol}$ of $3,0.6 \mathrm{mmol}$ of $\mathrm{ArB}(\mathrm{OH})_{2}, 1.6 \mathrm{mg}$ of [ $\left.\left\{\mathrm{Rh}\left(\mathrm{C}_{2} \mathrm{H}_{4}\right)_{2} \mathrm{Cl}\right\}_{2}\right](0.004 \mathrm{mmol}, 4.0 \mathrm{~mol} \% \mathrm{Rh}), 0.0096 \mathrm{mmol}$ of ligand $\mathbf{L 3 c}, 1$ $\mathrm{mL}$ of $\mathrm{MeOH}, 5.0 \mathrm{~mol} \%$ DIPEA $0.1 \mathrm{~mol} / \mathrm{L}$ in $\mathrm{MeOH}), 40{ }^{\circ} \mathrm{C}, 3 \mathrm{~h} .{ }^{b}$ Yield of the isolated product. ${ }^{c}$ Determined by HPLC analysis on a chiral stationary phase.

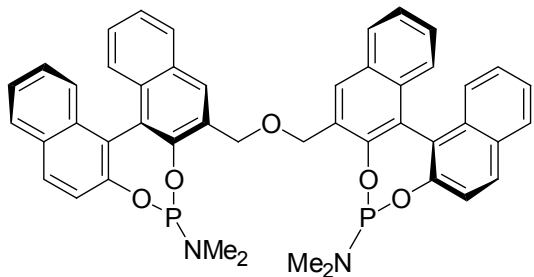

$(R, R)$-Me-BIPAM
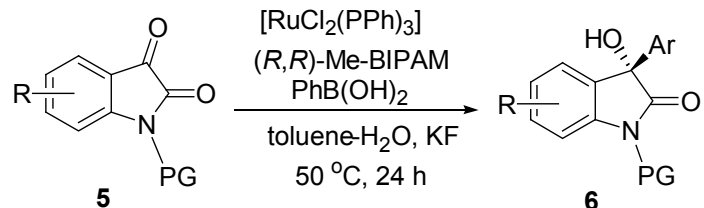

表 $3 \mathrm{Ru}$-催化苯基硼酸与靛红的不对称加成

Table 3 Ruthenium-catalyzed asymmetric addition of phenylboronic acids to isatins

\begin{tabular}{cccccl}
\hline Entry & $\mathrm{R}$ & $\mathrm{PG}$ & $\mathbf{6}$ & $\mathrm{Yield}^{a} / \%$ & $e e^{b} / \%$ \\
\hline 1 & $5-\mathrm{F}$ & $\mathrm{PMB}$ & $\mathbf{6 a}$ & $>99$ & 87 \\
2 & $5-\mathrm{F}$ & $\mathrm{Bn}$ & $\mathbf{6 b}$ & 95 & $90(R)$ \\
3 & $5-\mathrm{Br}$ & $\mathrm{PMB}$ & $\mathbf{6 c}$ & 97 & 90 \\
4 & $5-\mathrm{Br}$ & $\mathrm{Bn}$ & $\mathbf{6 d}$ & 96 & 86 \\
5 & $5-\mathrm{Me}$ & $\mathrm{PMB}$ & $\mathbf{6 e}$ & 97 & $90(R)$ \\
6 & $5-\mathrm{Me}$ & $\mathrm{Bn}$ & $\mathbf{6 f}$ & 92 & $87(R)$ \\
7 & $6-\mathrm{Cl}$ & $\mathrm{PMB}$ & $\mathbf{6 g}$ & 97 & 88 \\
8 & $6-\mathrm{Cl}$ & $\mathrm{Bn}$ & $\mathbf{6 h}$ & $>99$ & 89 \\
9 & $\mathrm{H}$ & $\mathrm{PMB}$ & $\mathbf{6 i}$ & $>99$ & $89(R)$ \\
\hline
\end{tabular}

${ }^{a}$ Isolated yields. ${ }^{b}$ Determined by HPLC.

作者认为反应的催化循环与 $\mathrm{Rh}$ 类似, 经历三个主 要步骤: (1) Ru/Me-BIPAM 与芳基硼酸发生转金属化得 到 $\mathrm{Ar}-\mathrm{Ru}{ }^{\mathrm{II}}$ 化合物; (2) $\mathrm{Ar}-\mathrm{Ru}{ }^{\mathrm{II}}$ 化合物与靛红进行插入 反应；(3) $\mathrm{O}-\mathrm{Ru}^{\mathrm{II}}$ 反应中间体在水的作用下得到加成产
物(图 3).

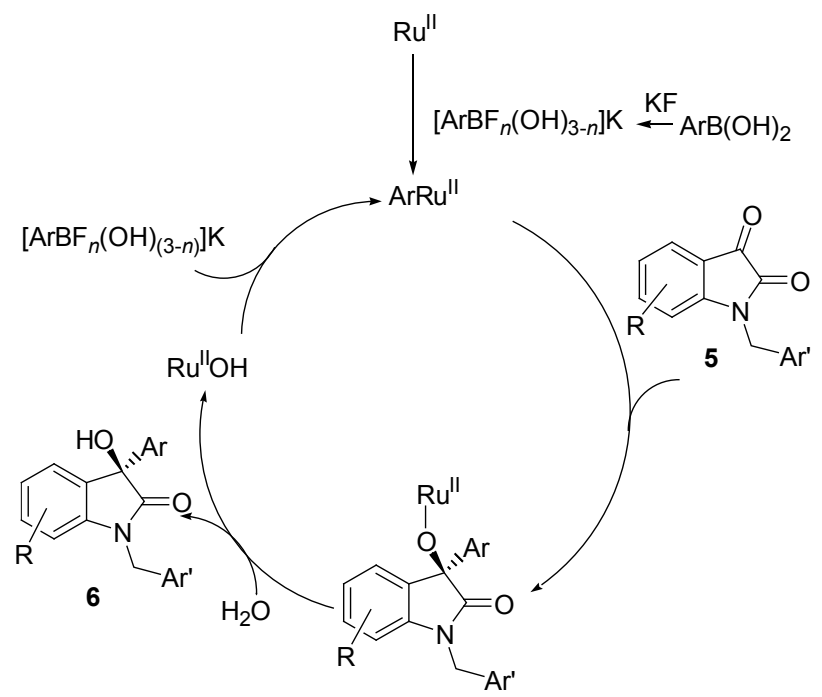

图 3 可能的催化循环

Figure 3 Proposed catalytic cycle

在过去的十年中, $\mathrm{N}$-杂环卡宾被看作是与膦配体相 对应的一类有效的配体已广泛应用于各类反应. 与膦配 体相比较该类配体具有更强的 $\delta$-给电子与较弱的 $\pi$ 重叠 作用，在特定的反应底物中与膦配体比较具有其独特的 优势. 此外, $\mathrm{N}$-杂环卡宾配体对空气湿气稳定等优点也 利于保存及使用. $\mathrm{Cu}-\mathrm{N}$-杂环卡宾化合物在许多羰基化 合物的加成反应中表现出很高的催化活性 ${ }^{[24]} .2009$ 年, Hayashi 等 ${ }^{[25]}$ 将手性的 $\mathrm{N}$-杂环卡宾配体 $\mathbf{L 4}$ 应用到芳基 硼酸酯与靛红的加成反应中(Eq. 4), 使用 $10.0 \mathrm{~mol} \%$ $\mathrm{CuCl}$ 与 $11.0 \mathrm{~mol} \% \mathrm{L4}, \mathrm{KO} t-\mathrm{Bu}$ 为碱, dioxane 为反应溶 剂, 在温和的条件下获得高收率与对映选择性的 3-着基 吲哚酮(表 4).

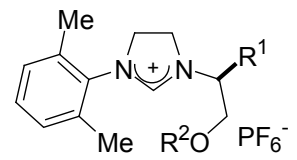

(S)-L4a: $\mathrm{R}^{1}=\mathrm{Ph}, \mathrm{R}^{2}=\mathrm{H}$

(S)-L4b: $\mathrm{R}^{1}=i-\mathrm{Pr}, \mathrm{R}^{2}=\mathrm{H}$

(S)-L4c: $R^{1}=t-B u, R^{2}=H$
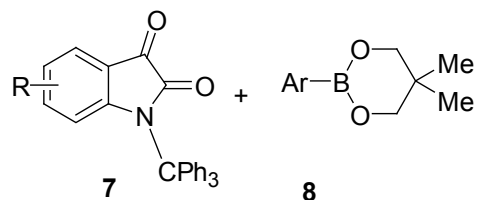

$\mathrm{CuCl}(10 \mathrm{~mol} \%)$

a: $\mathrm{R}=\mathrm{F} ; \mathbf{b}: \mathrm{R}=\mathrm{Me}$

c: $\mathrm{R}=\mathrm{Cl} ; \mathbf{d}: \mathrm{R}=\mathrm{Br}$

e: $\mathrm{R}=\mathrm{H}$

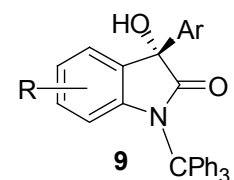


表 $4 \mathrm{Cu}$ 催化的芳基嗍酸酯 8 与靛红 7 的不对称加成

Table 4 Copper-catalyzed asymmetric addition of arylboronates 8 to isatins 7

\begin{tabular}{clccc}
\hline Entry & \multicolumn{1}{c}{$\mathbf{8}(\mathrm{Ar})$} & $\mathbf{9}$ & $\mathrm{Yield}^{a} / \%$ & $e e^{b} / \%$ \\
\hline 1 & $\mathbf{8 a}\left(4-\mathrm{MeOC}_{6} \mathrm{H}_{4}\right)$ & $\mathbf{9 a a}$ & 80 & 88 \\
2 & $\mathbf{8 a}$ & $\mathbf{9 b a}$ & 67 & 85 \\
3 & $\mathbf{8 a}$ & $\mathbf{9 c a}$ & 83 & 89 \\
4 & $\mathbf{8 a}$ & $\mathbf{9 d a}$ & 70 & 89 \\
$5^{\mathbf{c}}$ & $\mathbf{8 a}$ & $\mathbf{9 e a}$ & 76 & 85 \\
6 & $\mathbf{8 b}$ & $\mathbf{9 a b}$ & 74 & 91 \\
7 & $\mathbf{8 b}$ & $\mathbf{9 c b}$ & 83 & 92 \\
8 & $\mathbf{8 b}$ & $\mathbf{9 d b}$ & 87 & 92 \\
9 & $\mathbf{8 c}\left(4-\mathrm{MeC}_{6} \mathrm{H}_{4}\right)$ & $\mathbf{9 d c}$ & 87 & 92 \\
10 & $\mathbf{8 d}\left(4-\mathrm{ClC}_{6} \mathrm{H}_{4}\right)$ & $\mathbf{9 a d}$ & 78 & 86 \\
11 & $\mathbf{8 e}(2-\mathrm{Naphthyl})$ & $\mathbf{9 a e}$ & 85 & 86 \\
12 & $\mathbf{8 f}(3-\mathrm{Thienyl})$ & $\mathbf{9 a f}$ & 94 & 87 \\
13 & $\mathbf{8 g}\left(2-\mathrm{MeC}_{6} \mathrm{H}_{4}\right)$ & $\mathbf{9 a g}$ & 74 & 68 \\
14 & $\mathbf{8 h}(1-\mathrm{Cyclohexenyl})$ & $\mathbf{9 a h}$ & 72 & 67 \\
\hline
\end{tabular}

${ }^{a}$ Isolated yield. ${ }^{b}$ Determined by HPLC with chiral OD-H column. ${ }^{c}$ The reaction conducted for $36 \mathrm{~h}$.

作者认为反应的可能机理首先是 $\mathrm{CuCl}$ 与手性 $\mathrm{N}$-杂 环卡宾在碱的作用下生成叔丁醇氧 $\mathrm{N}$-杂环卡宾铜催化 剂, 接着与硼酸酯发生转金属化得到芳基铜化合物 $\mathbf{A}$, 然后与底物发生插入反应获得烷氧基铜化合物 $\mathbf{B}$, 最后 $\mathbf{B}$ 与碱发生配体交换获得加成产物前提 $\mathbf{C}$ 同时完成催化 剂循环, 化合物 $\mathbf{C}$ 在水的作用下转化成加成产物(图 4).

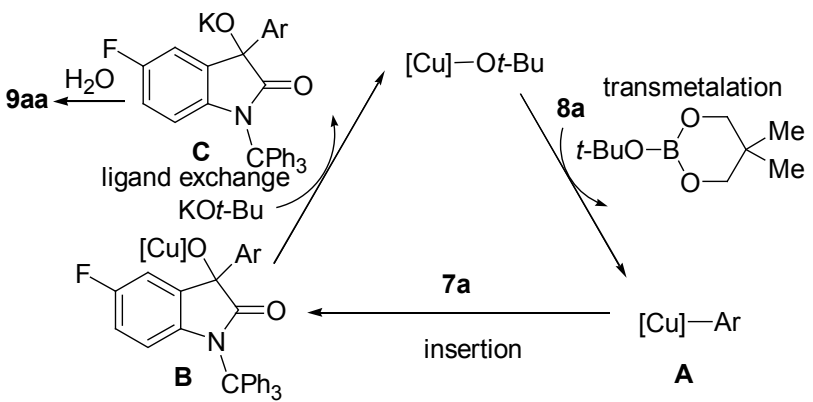

图 $4 \mathrm{Cu}$ 催化 $8 \mathbf{a}$ 与 $7 \mathbf{a}$ 加成的可能机理

Figure 4 Proposed catalytic cycle for the copper-catalyzed addition of $\mathbf{8 a}$ to $7 \mathbf{a}$

2011 年, Shi 等 ${ }^{[26]}$ 则报道了使用钯 $\mathrm{N}$-杂环卡宾二水 化合物 $(\mathrm{NHC}) \mathrm{Pd}^{2+}\left(\mathrm{OH}_{2}\right)_{2}(\mathrm{OTf})_{2}(\mathbf{1 2})$ 为催化剂不对称催 化芳基硼酸与青定红的加成(Eq. 5). 作者发现碱在反应中 的影响比较大, 在篎选不同碱后发现加入 $\mathrm{LiOH}$ 一水化 合物可较好的提高反应的催化活性, 使用 $5.0 \mathrm{~mol} \% \mathbf{1 2}$ 室温下反应获得了 79\% 94\%收率, 不足的是反应只获 得了中等偏上的对映选择性.

此外, 作者还提出了该反应可能的催化机理. 作者 认为反应中原位生成的 $\mathrm{LiOAr}$ 在反应速度上起着关键 的作用, 其主要原因是由于生成的 $\mathrm{LiOAr}$ 与反应底物作

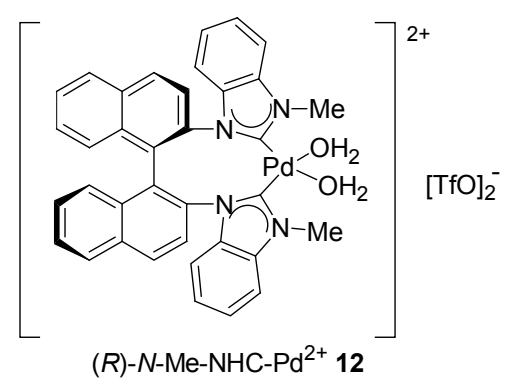

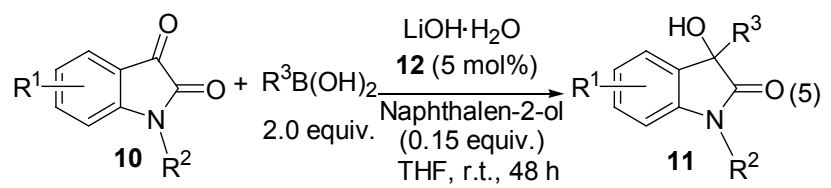

用从而活化了反应底物. 在此基础上作者提出了具体的 催化循环：首先正离子 $\mathrm{NHC}-\mathrm{Pd}^{2+}$ 二水化合物在碱的作 用下生成 $\mathrm{PdOH}$ 化合物 $\mathbf{D}$, 接着 $\mathbf{D}$ 与芳基硼酸发生转金 属化生成钯硼烷化合物 $\mathbf{E}, \mathbf{E}$ 发生 $\beta$-芳基消除得到芳基 化合物 $\mathbf{F}$ ，同时底物与原位生成的 LiOAr 形成反应中间 体 $\mathbf{G}, \mathbf{F}$ 与反应中间体 $\mathbf{G}$ 发生芳基化反应并在水的作用 下得到加成产物 11 同时催化剂再生(图 5).

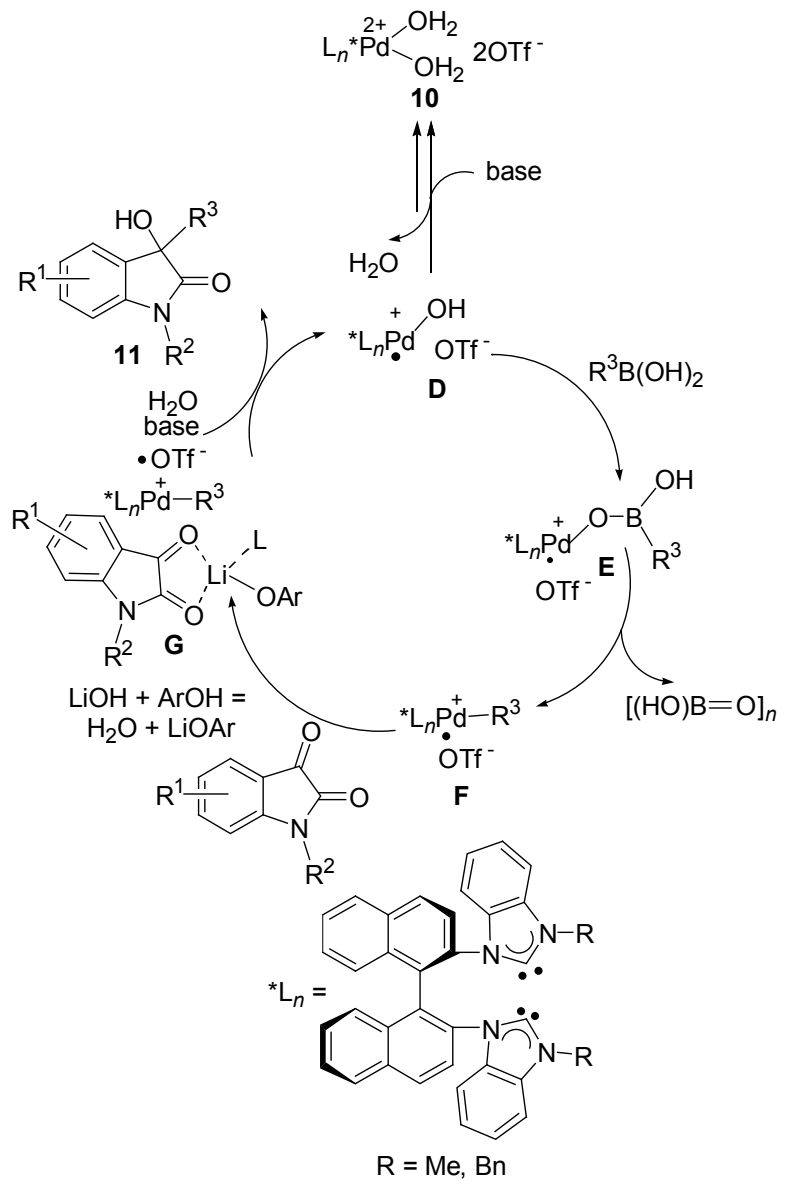

图 5 可能的反应机理

Figure 5 A plausible reaction mechanism 


\section{2 三氟甲基酮}

氟化合物由于其独特的性质已被广泛用于材料、医 药及农业化学等领域. 三氟甲基取代基的氟化合物常常 具有其独特的生物活性而成为氟化合物里重要的一类, 因此该类化合物的合成是一项非常有意义的研究工作. 目前该类化合物的合成方法主要有两种：一是对羰基化 合物进行三氟甲基化, 然而该方法的不对称合成往往难 以实现; 二是碳亲核试剂对三氟苯乙酮的加成, 金属催 化芳基硼酸与三氟苯乙酮就是合成三氟甲基化合物的 有效方法之一. 2006 年, Feringa 与 Minnard 等 ${ }^{[27]}$ 报道了 $\mathrm{Rh}$ 与手性亚磷酰胺(图 6)催化芳基硼酸与三氟苯乙酮的 不对称加成反应，以 MTBE 为反应溶剂，回流条件下反 应 $16 \mathrm{~h}$ 后可获得 28\% 94\%收率与 50\% 83\% ee. 反应 结果虽不理想, 但也为该类化合物的合成提供了很有效 的借鉴作用. 该部分研究工作在 2009 年 Wiley 出版的 《Modern Arylation Methods》一书中已有提及, 在此不再 赘述 ${ }^{[19]} .2011$ 年, Iulinano 等 ${ }^{[28]}$ 发现使用 $(S)$-联萗亚磷酸 化合物 $\mathbf{L 6}$ (图 6)与 $\left[\mathrm{RhCl}\left(\mathrm{C}_{2} \mathrm{H}_{4}\right)_{2}\right]_{2}$ 也可很好的催化芳基硼 酸与三氟苯乙䣶的加成，室温下使用 $0.75 \mathrm{~mol} \%$ $\left[\mathrm{RhCl}\left(\mathrm{C}_{2} \mathrm{H}_{4}\right)_{2}\right]_{2}$ 反应即可在较短的时间内完成并获得 $70 \% \sim 93 \%$ 收率，遗憾的是反应的对映选择性很不理想.

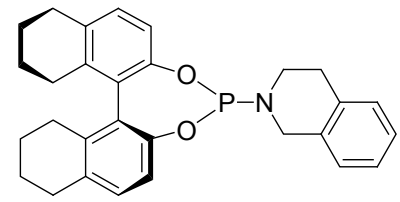

(S)-L5

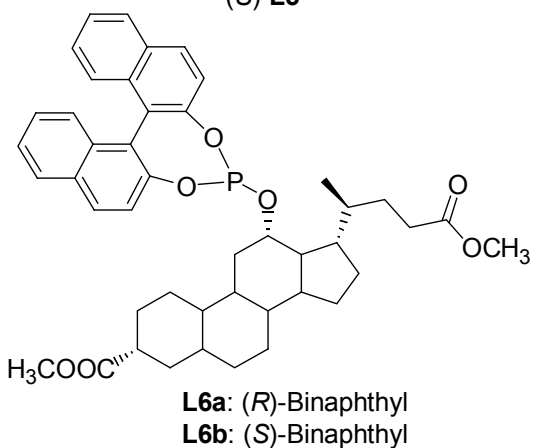

图 6 联萘亚磷酸配体 L6

Figure 6 Binaphthyl phosphite ligand L6

最近，作者所在的汤文军课题组 ${ }^{[29]}$ 报道使用手性 双膦配体 $\mathbf{L} 7$ 与 $\left[\mathrm{RhCl}\left(\mathrm{C}_{2} \mathrm{H}_{4}\right)_{2}\right]_{2}$ 催化芳基硼酸与三氟苯乙 酮的不对称加成反应(Eq. 6), 反应以甲基叔丁基醚 (MTBE) 为溶剂，回流条件下可获得高达 $99 \%$ ee 的加成 产物. 作者通过对产物进行 X单晶衍生确定了产物的绝 对构型为 $S$. 这是目前该反应获得的最好的不对称反应 结果(表 5).

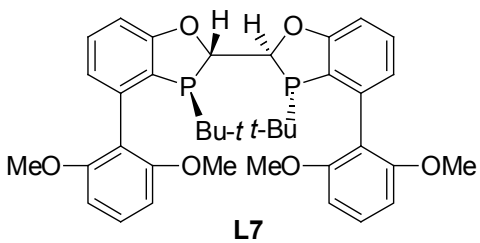

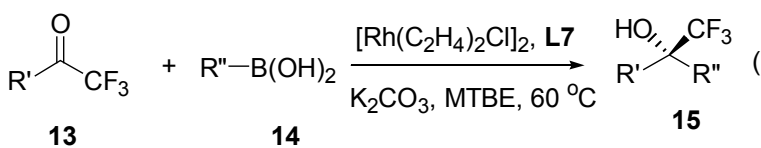

表 5 芳基嗍酸与三氟甲基苯乙酮的反应结果 ${ }^{a}$

Table 5 Addition of arylboronic acids to trifloromethyl methyl ketones

\begin{tabular}{|c|c|c|c|c|}
\hline Entry & $\mathrm{R}^{\prime}$ & $\mathrm{R}^{\prime \prime}$ & $\begin{array}{l}\text { Yield }^{b} \% \\
\text { (Compd.) }\end{array}$ & $e e^{c} / \%$ \\
\hline 1 & $\mathrm{Ph}$ & 4- $\mathrm{MeOC}_{6} \mathrm{H}_{4}$ & 79 (15a) & 95 \\
\hline 2 & $\mathrm{Ph}$ & $4-\mathrm{MeC}_{6} \mathrm{H}_{4}$ & $78(\mathbf{1 5 b})$ & 99 \\
\hline 3 & $\mathrm{Ph}$ & 4- $\mathrm{ClC}_{6} \mathrm{H}_{4}$ & 83 (15c) & 97 \\
\hline 4 & $\mathrm{Ph}$ & 4- $\mathrm{PhC}_{6} \mathrm{H}_{4}$ & $71(\mathbf{1 5 d})$ & 95 \\
\hline 5 & $\mathrm{Ph}$ & $3-\mathrm{MeOC}_{6} \mathrm{H}_{4}$ & 83 (15e) & 95 \\
\hline 6 & 4- $-\mathrm{FC}_{6} \mathrm{H}_{4}$ & $4-\mathrm{MeOC}_{6} \mathrm{H}_{4}$ & 81 (15f) & 95 \\
\hline 7 & $4-\mathrm{FC}_{6} \mathrm{H}_{4}$ & $\mathrm{Ph}$ & $61(\mathbf{1 5 g})$ & 95 \\
\hline 8 & $4-\mathrm{FC}_{6} \mathrm{H}_{4}$ & 4- $\mathrm{MeC}_{6} \mathrm{H}_{4}$ & 85 (15h) & 97 \\
\hline 9 & $4-\mathrm{FC}_{6} \mathrm{H}_{4}$ & 4- $\mathrm{ClC}_{6} \mathrm{H}_{4}$ & $86(\mathbf{1 5 i})$ & 97 \\
\hline 10 & $4-\mathrm{FC}_{6} \mathrm{H}_{4}$ & 4- $\mathrm{PhC}_{6} \mathrm{H}_{4}$ & $73(\mathbf{1 5} \mathbf{j})$ & 97 \\
\hline 11 & $4-\mathrm{FC}_{6} \mathrm{H}_{4}$ & $4-\mathrm{TMSC}_{6} \mathrm{H}_{4}$ & 91 (15k) & 98 \\
\hline 12 & $4-\mathrm{FC}_{6} \mathrm{H}_{4}$ & $4-\mathrm{BuC}_{6} \mathrm{H}_{4}$ & 91 (15l) & 96 \\
\hline 13 & $4-\mathrm{FC}_{6} \mathrm{H}_{4}$ & $4-i-\mathrm{PrC}_{6} \mathrm{H}_{4}$ & 93 (15m) & 97 \\
\hline 14 & 4- $-\mathrm{FC}_{6} \mathrm{H}_{4}$ & $3-\mathrm{CF}_{3} \mathrm{OC}_{6} \mathrm{H}_{4}$ & 87 (15n) & 99 \\
\hline 15 & 4- $-\mathrm{FC}_{6} \mathrm{H}_{4}$ & 2-Naphthyl & $61(\mathbf{1 5 o})$ & 95 \\
\hline 16 & $4-\mathrm{FC}_{6} \mathrm{H}_{4}$ & $2-\mathrm{FC}_{6} \mathrm{H}_{4}$ & 73 (15p) & 99 \\
\hline 17 & 4- $\mathrm{FC}_{6} \mathrm{H}_{4}$ & $2-\mathrm{MeOC}_{6} \mathrm{H}_{4}$ & $37(\mathbf{1 5 q})$ & 81 \\
\hline 18 & $4-\mathrm{ClC}_{6} \mathrm{H}_{4}$ & 4- $\mathrm{MeOC}_{6} \mathrm{H}_{4}$ & $83(\mathbf{1 5 r})$ & 93 \\
\hline 19 & $4-\mathrm{ClC}_{6} \mathrm{H}_{4}$ & $4-\mathrm{MeC}_{6} \mathrm{H}_{4}$ & $86(\mathbf{1 5 s})$ & 96 \\
\hline 20 & 4- $\mathrm{BrC}_{6} \mathrm{H}_{4}$ & $4-\mathrm{MeOC}_{6} \mathrm{H}_{4}$ & $81(\mathbf{1 5 t})$ & 95 \\
\hline 21 & $4-\mathrm{BrC}_{6} \mathrm{H}_{4}$ & 4- $\mathrm{MeC}_{6} \mathrm{H}_{4}$ & $81(\mathbf{1 5 u})$ & 95 \\
\hline 22 & 4- $\mathrm{BrC}_{6} \mathrm{H}_{4}$ & 4- $\mathrm{ClC}_{6} \mathrm{H}_{4}$ & $83(15 v)$ & 96 \\
\hline 23 & 4- $\mathrm{MeC}_{6} \mathrm{H}_{4}$ & $4-\mathrm{MeOC}_{6} \mathrm{H}_{4}$ & $47(\mathbf{1 5 w})$ & 93 \\
\hline 24 & 4- $\mathrm{MeC}_{6} \mathrm{H}_{4}$ & $4-i-\mathrm{PrC}_{6} \mathrm{H}_{4}$ & $58(\mathbf{1 5 x})$ & 96 \\
\hline 25 & Thienyl & 4- $\mathrm{MeOC}_{6} \mathrm{H}_{4}$ & 81 (15y) & 81 \\
\hline 26 & Thienyl & 4- $\mathrm{ClC}_{6} \mathrm{H}_{4}$ & $61(\mathbf{1 5 z})$ & 89 \\
\hline 27 & $2-\mathrm{MeOC}_{6} \mathrm{H}_{4}$ & $\mathrm{Ph}$ & 31 (15aa) & 96 \\
\hline 28 & $3-\mathrm{CF}_{3} \mathrm{OC}_{6} \mathrm{H}_{4}$ & $4-\mathrm{MeOC}_{6} \mathrm{H}_{4}$ & 79 (15ab) & 96 \\
\hline
\end{tabular}

${ }^{a}$ The reactions were carried out at $60{ }^{\circ} \mathrm{C}$ in MTBE $(1.5 \mathrm{~mL})$ for $20 \mathrm{~h}$ with ketone $(0.25 \mathrm{mmol})$, boronic acid $(0.5 \mathrm{mmol})$, and $\mathrm{K}_{2} \mathrm{CO}_{3}(0.75 \mathrm{mmol})$ in the presence of $\left[\mathrm{Rh}_{(}\left(\mathrm{C}_{2} \mathrm{H}_{4}\right) \mathrm{Cl}\right]_{2}(1.5 \mathrm{~mol} \%)$ and ligand $\mathbf{L} 7(3.6 \mathrm{~mol} \%) ;{ }^{b}$ Isolated yields. ${ }^{c}$ Determined by chiral HPLC on a Chiralcel OD-H, Chiralcel AD-H, or Lux Amylose-2 column.

根据实验结果及催化剂与产物的绝对构型，作者还 提出了该催化反应可能的反应机理(图 7): 在碱性条件 下， $\left[\mathrm{Rh}\left(\mathrm{CH}_{2} \mathrm{CH}_{2}\right)_{2} \mathrm{Cl}\right]_{2}$ 与手性双膦配体转变成羟基铑络 合物 17, 铑化合物 17 与芳基硼酸 14 作用得到铑化合物 18, 然后与底物 13 作用配位得到两种可能的反应过渡 态 $\mathbf{H}$ 与 I. 在 I 过渡态中由于三氟甲基与手性膦配体的 
位阻作用使得该过渡态不稳定, 相反在 $\mathbf{H}$ 过渡态中由于 其较小的位阻使得其较为稳定, 易于进行插入反应及转 金属化从而获得选择性的加成产物. 该可能的反应机理 所得到产物的构型与经 $\mathrm{X}$ 单晶衍射得到的产物构型相 吻合.
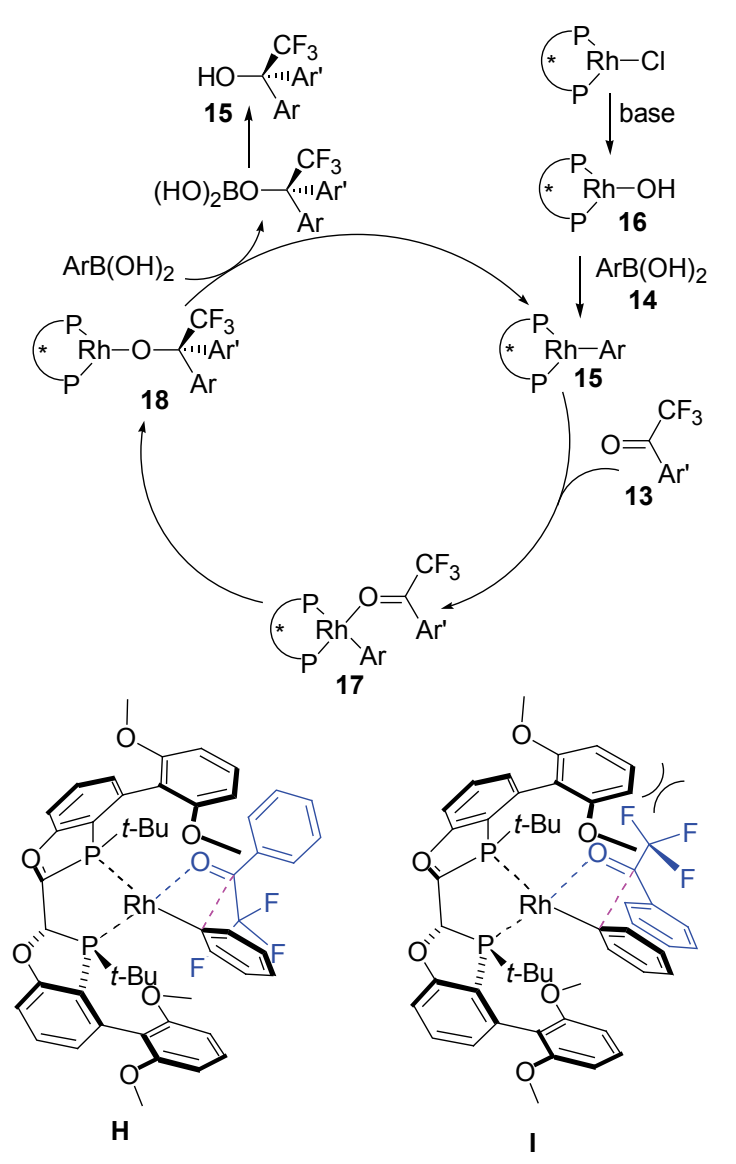

图 7 催化反应过程中可能的立体模型

Figure 7 The stereochemical model of the Rh-L7 catalyst during addition of arylboronic acids to trifluoromethyl ketones

\section{3 a-酮酯}

金属催化芳基嗍酸与芳基酮酯的不对称加成可获 得手性 2-羊基二芳基酸酯化合物, 该类化合物是有效的 毒苔碱受体拮抗剂 ${ }^{[30]} .2007$ 年, $\mathrm{Hu}$ 等 ${ }^{[31]}$ 报道使用亚磷酸 酯或二茂铁钯化合物催化芳基硼酸与酮酯的加成可获 得很高的反应收率, 遗憾的是只获得消旋的加成产物. 手性螺环膦配体是 Zhou 设计合成的一类新型膦配体, 在许多催化反应尤其是不对称氢化反应中表现出极好 的活性 ${ }^{[32]}$. 2008 年, 该小组 ${ }^{[33]}$ 第一次将该类手性配体应 用到芳基硼酸与酩酯的不对称加成中(Eq. 7), 研究发现 $3.0 \mathrm{~mol} \%\left[\mathrm{RhCl}\left(\mathrm{C}_{2} \mathrm{H}_{4}\right)_{2}\right]_{2}$ 与 $6.0 \mathrm{~mol} \% \mathbf{L 8}$, 以 $\mathrm{KF}$ 为碱, $1,2-$ 二氯乙烷(DCE) 与水为混合溶剂, 室温下反应可获 得很好的反应结果(表 6).

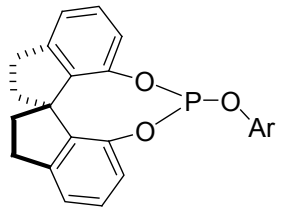

(S)-ShiP L8

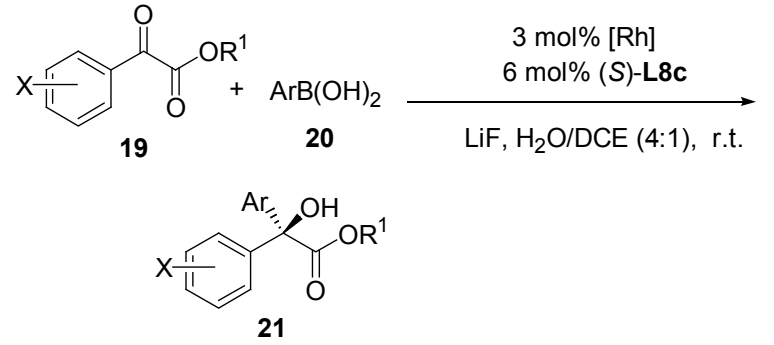

表 $6 \mathrm{Rh} /(S)$-L8c 催化芳基硼酸 20 与 $\alpha$-酮酯 19 的不对称加 成 ${ }^{a}$

Table 6 Asymmetric addition of arylboronic acid 20 to $\alpha$-ketoesters 19 catalyzed by $\mathrm{Rh} /(S)$-L8c

\begin{tabular}{|c|c|c|c|c|c|}
\hline Entry & $\mathrm{R}^{1}$ & $\mathrm{X}$ & $\mathrm{Ar}$ & Yield $^{b} / \%$ & $e e^{c} / \%$ \\
\hline 1 & $\mathrm{Et}$ & $4-\mathrm{Cl}$ & $\mathrm{C}_{6} \mathrm{H}_{5}$ & 96 & 77 \\
\hline 2 & $i$-Pr & $4-\mathrm{Cl}$ & $\mathrm{C}_{6} \mathrm{H}_{5}$ & 93 & 80 \\
\hline 3 & $t-\mathrm{Bu}$ & $4-\mathrm{Cl}$ & $\mathrm{C}_{6} \mathrm{H}_{5}$ & 51 & 70 \\
\hline 4 & $\mathrm{Ph}$ & $4-\mathrm{Cl}$ & $\mathrm{C}_{6} \mathrm{H}_{5}$ & 90 & 72 \\
\hline 5 & $\mathrm{Bn}$ & $4-\mathrm{Cl}$ & $\mathrm{C}_{6} \mathrm{H}_{5}$ & 95 & 84 \\
\hline 6 & $\mathrm{Bn}$ & $4-\mathrm{Cl}$ & $\mathrm{C}_{6} \mathrm{H}_{5}$ & 81 & 90 \\
\hline 7 & $\mathrm{Bn}$ & $4-\mathrm{F}$ & $\mathrm{C}_{6} \mathrm{H}_{5}$ & 84 & 88 \\
\hline 8 & $\mathrm{Bn}$ & $4-\mathrm{CF}_{3}$ & $\mathrm{C}_{6} \mathrm{H}_{5}$ & 93 & 91 \\
\hline 9 & $\mathrm{Bn}$ & 4-Me & $\mathrm{C}_{6} \mathrm{H}_{5}$ & 96 & 84 \\
\hline 10 & $\mathrm{Bn}$ & $4-\mathrm{MeO}$ & $\mathrm{C}_{6} \mathrm{H}_{5}$ & 93 & 86 \\
\hline 11 & $\mathrm{Bn}$ & $3,4-(\mathrm{CH})_{4}$ & $\mathrm{C}_{6} \mathrm{H}_{5}$ & 78 & 80 \\
\hline 12 & $\mathrm{Bn}$ & $\mathrm{H}$ & 4- $\mathrm{MeC}_{6} \mathrm{H}_{4}$ & 85 & 80 \\
\hline 13 & $\mathrm{Bn}$ & $\mathrm{H}$ & $4-\mathrm{MeOC}_{6} \mathrm{H}_{4}$ & 80 & 83 \\
\hline
\end{tabular}

此外，该反应催化体系对不饱和烯酮酯也有很好的 催化效果(Eq. 8). 反应中使用不同取代基芳基及杂环硼 酸均可获得很好的结果(表 7).
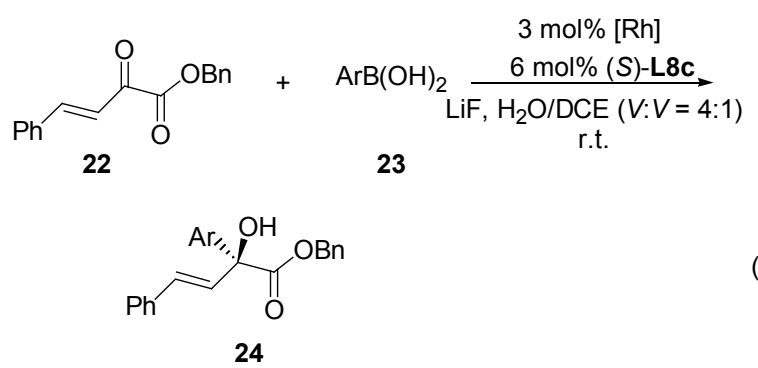

2011 年, Ready 等 ${ }^{[34]}$ 设计合成了手性丙二烯双膦配 体并将其用于催化芳基嗍酸与芳基酩酯的不对称加成 反应(Eq. 9), 以 $1.0 \mathrm{~mol} \%\left[\mathrm{RhCl}\left(\mathrm{C}_{2} \mathrm{H}_{4}\right)_{2}\right]_{2}$ 与 $2.0 \mathrm{~mol} \% \mathbf{~ L 9}$ 为催化剂反应可获得 $52 \%$ ～98\%收率与 79\% 95\% ee, 反应对芳基硼酸的效果较好，如使用烯基硼酸只获得中 
表 $7 \mathrm{Rh} /(S)$-L8c 催化芳基硼酸 $\mathbf{2 3}$ 与苯基乙烯酮酯 22 的不对 称加成 ${ }^{a}$

Table 7 Asymmetric addition of aryboronic acids 23 to benzyl 2-phenyl-vinyl-2-ketoesters 22 catalyzed by $\mathrm{Rh} /(S)$-L8c

\begin{tabular}{clccc}
\hline Entry & \multicolumn{1}{c}{$\mathrm{Ar}$} & $\mathbf{2 4}$ & $\mathrm{Yield}^{b} / \%$ & $e e^{c} / \%$ \\
\hline $1^{d}$ & $\mathrm{C}_{6} \mathrm{H}_{5}$ & $\mathbf{2 4 a}$ & 70 & 93 \\
$2^{d}$ & $4-\mathrm{MeC}_{6} \mathrm{H}_{4}$ & $\mathbf{2 4 b}$ & 77 & 93 \\
$3^{d}$ & $4-\mathrm{MeOC}_{6} \mathrm{H}_{4}$ & $\mathbf{2 4 c}$ & 75 & 90 \\
4 & $4-\mathrm{FC}_{6} \mathrm{H}_{5}$ & $\mathbf{2 4 d}$ & 93 & 92 \\
5 & $4-\mathrm{CF}_{3} \mathrm{C}_{6} \mathrm{H}_{4}$ & $\mathbf{2 4 e}$ & 61 & 90 \\
$6^{d}$ & $3-\mathrm{MeC}_{6} \mathrm{H}_{4}$ & $\mathbf{2 4 f}$ & 75 & 91 \\
$7^{d}$ & $3-\mathrm{MeOC}_{6} \mathrm{H}_{4}$ & $\mathbf{2 4 g}$ & 70 & 93 \\
8 & $2-\mathrm{MeC}_{6} \mathrm{H}_{4}$ & $\mathbf{2 4 h}$ & 92 & 75 \\
9 & $2-\mathrm{Naphthyl}$ & $\mathbf{2 4 i}$ & 89 & 90 \\
10 & $2-\mathrm{Thienyl}$ & $\mathbf{2 4 j}$ & 91 & 88 \\
\hline${ }^{a} \mathbf{2 2} / \mathbf{2 3} / \mathrm{LiF} /\left\{\mathrm{RhCl}\left(\mathrm{CH}_{2} \mathrm{CH}_{2}\right)_{2}\right\}_{2} /$ ligand $=1: 2: 2: 0.015: 0.06 .{ }^{b}$ Yield of
\end{tabular}

isolated product. ${ }^{c}$ Determined bu chiral HPLC analysis. ${ }^{d} 0{ }^{\circ} \mathrm{C}$.

等偏上的对映选择性(表 8$)$.

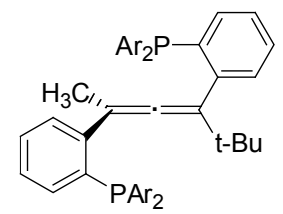

L9 $\mathrm{Ar}=3,5-\left(\mathrm{CF}_{3}\right)_{2}-\mathrm{C}_{6} \mathrm{H}_{3}$
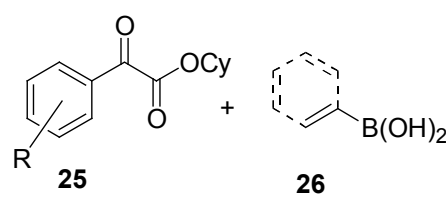

$\left[\mathrm{Rh}\left(\mathrm{C}_{2} \mathrm{H}_{4}\right)_{2} \mathrm{Cl}\right]_{2}(1 \mathrm{~mol} \%)$

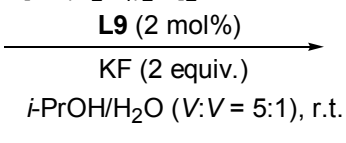<smiles>[R]c1ccc(C(O)(C(=O)OCCC)c2ccccc2)cc1</smiles>

作者同时也合成分离出金属 $\mathrm{Rh}$ 与 $\mathbf{L 9}$ 的络合物并 获得其单晶构型, 通过单晶衍射发现金属 $\mathrm{Rh}$ 与手性丙 二烯双膦配体进行膦烯三齿配位(图 8), 该类配位方式 更有利于催化剂的稳定性从而提高催化剂的催化活性. 手性配体上双膦取代基的电负性对催化活性也有较大 的影响，强吸电子的 3,5-二三氟甲基苯基取代基配体的 活性最好, 可能的原因是催化剂与底物络合后通过诱导 效应可有效减弱反应底物的电负性使得反应更易进行.

前面提到 Yamamoto 等 ${ }^{[22 b]}$ 使用 $(R, R)-\mathrm{Me}-\mathrm{BIPAM}$ 与 $\mathrm{Ru}$ 可很好的催化芳基硼酸与靛红的不对称加成，随后 该小组发现该配体也可很好的催化酩酯的不对称加成, 反应不仅可很好的催化芳基取代酮酯，对烷基取代酮酯 也可获得很理想的反应结果.
表 $8 \mathrm{Rh}$ 催化的芳基嗍酸与 $\alpha$-酮酯的不对称加成 ${ }^{a}$

Table 8 Enantioselective $\mathrm{Rh}(\mathrm{I})$-catalyzed addition of boronic acids to $\alpha$-keto esters: reaction scope

\begin{tabular}{|c|c|c|c|c|c|}
\hline Entry & $\mathrm{R}^{1}$ & $\mathrm{Ar}$ & Time/h & Yield $^{b} / \%$ & $e e^{c} / \%$ \\
\hline $1^{c}$ & $\mathrm{H}$ & $4-\mathrm{MeOC}_{6} \mathrm{H}_{4}$ & 12 & 90 & 91 \\
\hline 2 & $\mathrm{H}$ & 4- $\mathrm{PhC}_{6} \mathrm{H}_{4}$ & 60 & 88 & 88 \\
\hline 3 & $\mathrm{H}$ & 2-Naphthyl & 60 & 74 & 87 \\
\hline 4 & $3,5-\left(\mathrm{CF}_{3}\right)_{2}$ & $4-\mathrm{MeOC}_{6} \mathrm{H}_{4}$ & 6 & 90 & 79 \\
\hline 5 & $3,5-\left(\mathrm{CF}_{3}\right)_{2}$ & $\mathrm{Ph}$ & 6 & 89 & 91 \\
\hline 6 & $3,5-\left(\mathrm{CF}_{3}\right)_{2}$ & 4- $\mathrm{PhC}_{6} \mathrm{H}_{4}$ & 6 & 94 & 89 \\
\hline 7 & $3,5-\left(\mathrm{CF}_{3}\right)_{2}$ & 2-Naphthyl & 6 & 90 & 90 \\
\hline 8 & $3,5-\left(\mathrm{CF}_{3}\right)_{2}$ & $3-\mathrm{MeOC}_{6} \mathrm{H}_{4}$ & 6 & 87 & 91 \\
\hline 9 & $3,5-\left(\mathrm{CF}_{3}\right)_{2}$ & $4-\mathrm{ClC}_{6} \mathrm{H}_{4}$ & 6 & 80 & 91 \\
\hline 10 & $4-\mathrm{CN}$ & $4-\mathrm{MeOC}_{6} \mathrm{H}_{4}$ & 6 & 95 & 91 \\
\hline 11 & $4-\mathrm{CN}$ & $\mathrm{Ph}$ & 6 & 81 & 95 \\
\hline 12 & $4-\mathrm{CN}$ & 4- $\mathrm{PhC}_{6} \mathrm{H}_{4}$ & 6 & 88 & 93 \\
\hline 13 & $4-\mathrm{CN}$ & 2-Naphthyl & 6 & 87 & 92 \\
\hline 14 & $4-\mathrm{CN}$ & $3-\mathrm{MeOC}_{6} \mathrm{H}_{4}$ & 6 & 93 & 94 \\
\hline 15 & $4-\mathrm{F}$ & 4- $\mathrm{PhC}_{6} \mathrm{H}_{4}$ & 48 & 77 & 92 \\
\hline 16 & $4-\mathrm{F}$ & 2-Naphthyl & 48 & 73 & 83 \\
\hline 17 & $4-\mathrm{Me}$ & $4-\mathrm{MeOC}_{6} \mathrm{H}_{4}$ & 48 & 81 & 84 \\
\hline 18 & $4-\mathrm{Me}$ & 4- $\mathrm{PhC}_{6} \mathrm{H}_{4}$ & 72 & 58 & 87 \\
\hline 19 & $4-\mathrm{MeO}$ & $4-\mathrm{Me}_{2} \mathrm{NC}_{6} \mathrm{H}_{4}$ & 20 & 98 & 83 \\
\hline $20^{d}$ & 4-MeO & $4-\mathrm{ClC}_{6} \mathrm{H}_{4}$ & 72 & 37 & 80 \\
\hline 21 & $4-\mathrm{Cl}$ & $4-\mathrm{MeOC}_{6} \mathrm{H}_{4}$ & 12 & 97 & 90 \\
\hline 22 & $4-\mathrm{Cl}$ & 4- $\mathrm{PhC}_{6} \mathrm{H}_{4}$ & 24 & 87 & 90 \\
\hline 23 & $3-\mathrm{Cl}$ & $4-\mathrm{MeOC}_{6} \mathrm{H}_{4}$ & 16 & 98 & 93 \\
\hline 24 & $3-\mathrm{Cl}$ & 2-Naphthyl & 40 & 97 & 86 \\
\hline 25 & $2-\mathrm{Cl}$ & $4-\mathrm{MeOC}_{6} \mathrm{H}_{4}$ & 72 & 84 & 92 \\
\hline 26 & $2-\mathrm{CF}_{3}$ & $4-\mathrm{MeOC}_{6} \mathrm{H}_{4}$ & 72 & 52 & 90 \\
\hline
\end{tabular}

${ }^{a}$ Conditions: $0.06 \mathrm{mmol}$ of $\mathbf{2 5}, 0.12 \mathrm{mmol}$ of $\mathbf{2 6}, 0.1 \mathrm{~mol} / \mathrm{L}$ under a $\mathrm{N}_{2}$ atmosphere, unless otherwise noted. ${ }^{b}$ Isolated yields. ${ }^{c} 2.6 \mathrm{mmol}$ of $25,5.2 \mathrm{mmol}$ of 26. ${ }^{d}$ Reaction conducted at $40{ }^{\circ} \mathrm{C}$.

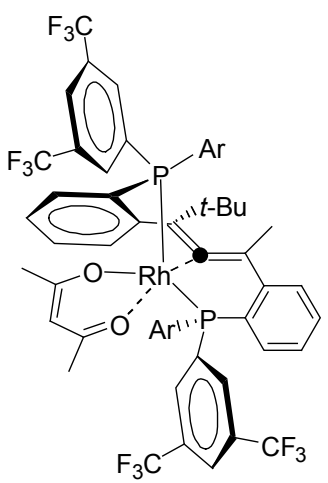

图 8 催化剂 $\left[\mathrm{RhCl}\left(\mathrm{C}_{2} \mathrm{H}_{4}\right)_{2}\right]_{2} / \mathbf{L 9}$ 的单晶结构

Figure 8 The X-ray of catalyst $\left[\mathrm{RhCl}\left(\mathrm{C}_{2} \mathrm{H}_{4}\right)_{2}\right]_{2} / \mathbf{L 9}$

手性烯烃亚磺酰胺在不对称芳基硼酸与不饱和羰 基化合物的 1,4-加成中是一类非常有效的催化配体 ${ }^{[35]}$. 最近, $\mathrm{Xu}$ 等 ${ }^{[36]}$ 报道了化合物 $\mathbf{L 1 0}$ 与 $\left[\left\{\mathrm{Rh}(\mathrm{coe})_{2} \mathrm{Cl}\right\}_{2}\right]$ 催化 芳基酩酯与芳基硼酸的加成反应 (Eq. 10), 以 $\mathrm{KOH}$ 为 碱，四氢呋喃(THF)为反应溶剂室温下反应可在较短时 间内完成并获得高收率与对映选择性的加成产物(表 9). 


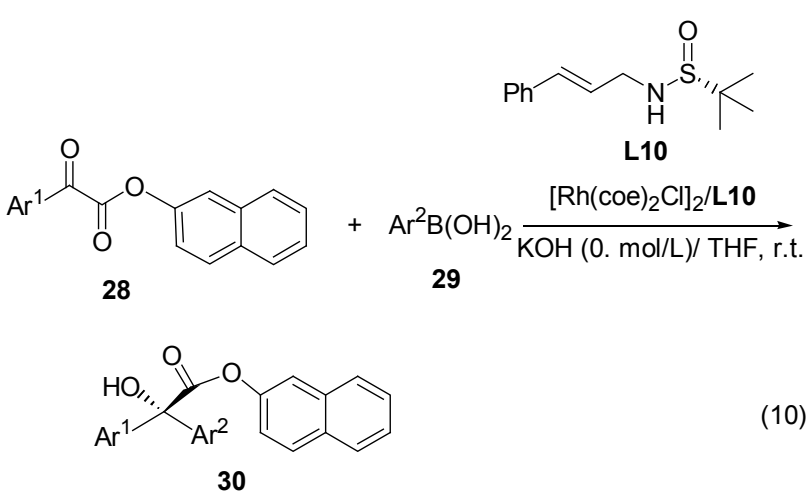

表 $9\left[\left\{\mathrm{Rh}(\mathrm{coe})_{2} \mathrm{Cl}\right\}_{2}\right] / \mathbf{L 1 0}$ 催化的芳基硼酸与 $\alpha$-酮酯的不对称 加成 ${ }^{a}$

Table 9 Asymmetric 1,2-addition of arylboronic acids to $\alpha$-ketoesters catalyzed by $\left[\left\{\mathrm{Rh}(\text { coe })_{2} \mathrm{Cl}\right\}_{2}\right] / \mathbf{L 1 0}$

\begin{tabular}{|c|c|c|c|c|}
\hline Entry & $\mathrm{Ar}^{1}$ & $\mathrm{Ar}^{2}$ & Yield $^{b} / \%$ & $e e^{c} / \%$ \\
\hline 1 & $\mathrm{Ph}$ & 4- $\mathrm{MeC}_{6} \mathrm{H}_{4}$ & 86 & 94 \\
\hline 2 & $\mathrm{Ph}$ & $4-\mathrm{ClC}_{6} \mathrm{H}_{4}$ & 84 & 94 \\
\hline 3 & $\mathrm{Ph}$ & 4- $\mathrm{FC}_{6} \mathrm{H}_{4}$ & 86 & 94 \\
\hline 4 & $\mathrm{Ph}$ & $4-\mathrm{MeOC}_{6} \mathrm{H}_{4}$ & 94 & 92 \\
\hline 5 & $\mathrm{Ph}$ & $2-\mathrm{MeC}_{6} \mathrm{H}_{4}$ & 72 & 92 \\
\hline 6 & $\mathrm{Ph}$ & $2-\mathrm{MeOC}_{6} \mathrm{H}_{4}$ & 73 & 90 \\
\hline 7 & $\mathrm{Ph}$ & $3-\mathrm{MeC}_{6} \mathrm{H}_{4}$ & 82 & 93 \\
\hline 8 & $\mathrm{Ph}$ & $3-\mathrm{ClC}_{6} \mathrm{H}_{4}$ & 67 & 95 \\
\hline 9 & $\mathrm{Ph}$ & $3-\mathrm{FC}_{6} \mathrm{H}_{4}$ & 72 & 95 \\
\hline 10 & $\mathrm{Ph}$ & $3-\mathrm{MeOC}_{6} \mathrm{H}_{4}$ & 72 & 93 \\
\hline 11 & $\mathrm{Ph}$ & $3,5-\mathrm{Me}_{2} \mathrm{C}_{6} \mathrm{H}_{3}$ & 74 & 92 \\
\hline 12 & $\mathrm{Ph}$ & 1-Naphthyl & 87 & 91 \\
\hline 13 & $\mathrm{Ph}$ & 2-Naphthyl & 76 & 92 \\
\hline 14 & $\mathrm{Ph}$ & $n-\mathrm{BuC}_{6} \mathrm{H}_{4}$ & 82 & 93 \\
\hline 15 & $4-\mathrm{ClC}_{6} \mathrm{H}_{4}$ & $\mathrm{Ph}$ & 66 & 95 \\
\hline 16 & $4-\mathrm{ClC}_{6} \mathrm{H}_{4}$ & 4- $\mathrm{MeC}_{6} \mathrm{H}_{4}$ & 66 & 92 \\
\hline 17 & $4-\mathrm{ClC}_{6} \mathrm{H}_{4}$ & $4-\mathrm{FC}_{6} \mathrm{H}_{4}$ & 70 & 95 \\
\hline 18 & $4-\mathrm{ClC}_{6} \mathrm{H}_{4}$ & 2-Naphthyl & 75 & 93 \\
\hline 19 & 2-Naphthyl & $\mathrm{Ph}$ & 89 & 91 \\
\hline 20 & 2-Naphthyl & $4-\mathrm{ClC}_{6} \mathrm{H}_{4}$ & 87 & 94 \\
\hline 21 & 2-Naphthyl & $2-\mathrm{MeC}_{6} \mathrm{H}_{4}$ & 54 & 93 \\
\hline
\end{tabular}

${ }^{a}$ Reactions were carried out with $\alpha$-ketoesters $(0.25 \mathrm{mmol})$ and 1.5 equiv. aryl boronic acid, in the presence of $3.0 \mathrm{~mol} \%[\mathrm{Rh}], 3.3 \mathrm{~mol} \%$ ligand L10, and $\mathrm{KOH}(0.1 \mathrm{~mol} / \mathrm{L}, 0.08$ equiv. $)$ in THF $(1.0 \mathrm{~mL})$ for $3 \mathrm{~h} .{ }^{b}$ Isolated yield. ${ }^{\mathrm{c}}$ Determined by HPLC.

\section{1,2-二酮}

邻羰基叔醇化合物是合成烟酸受体激动剂呋喃 酸 ${ }^{[37]}$ 与 HIV 整合酶抑制剂 Integrastatin $A$ 或 $\mathrm{B}^{[38]}$ 的重要 前体. 以往合成该类化合物的主要方法一般使用 Friedel-Crafts 反应或使用有机锡试剂为亲核试剂, 但 Friedel-Crafts 反应对富电子芳香底物结果的不理想及有 机锡试剂的毒性往往限制了该类合成方法的应用, 金属 催化芳基硼酸与 $1,2-$ 二酮化合物的不对称加成反应是合 成该类化合物较为理想的方法. 2007 年, Chisholm ${ }^{[39]}$ 报 道了 $\mathrm{Rh}(\mathrm{acac})(\mathrm{CO})_{2}$ 与 $\mathrm{PPh}(\mathrm{Cy})_{2}$ 为催化剂催化芳基硼酸
与 $1,2-$ 二酮化合物的加成, 遗憾的是反应只获得外消旋 的加成产物. 直至最近徐明华课题组发现 ${ }^{[36]}$ 研究发现 使用手性烯烃-亚磺酸胺配体 L10 不仅可以很好的催化 芳基硼酸与 $\alpha$-酮酯的不对称加成, 对 1,2-二酮也可获得 很好的获得很好的催化结果(表 10). 反应对于二芳基二 酮均可获得大于 $95 \%$ 的对映选择性，不足的是对脂肪族 二酮反应的对映选择性有明显的降低.

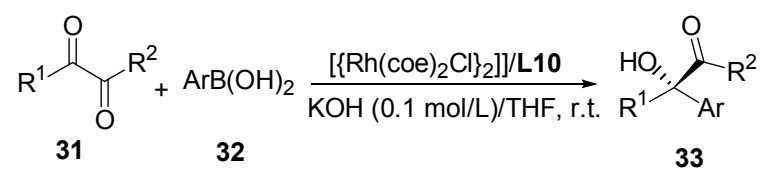

表 $10\left[\left\{\mathrm{Rh}(\mathrm{coe}){ }_{2} \mathrm{Cl}\right\}_{2}\right] / \mathbf{L 1 0}$ 催化芳基嗍酸与 $\alpha$ 二二酮的不对称 1,2 加成 ${ }^{a}$

Table 10 Asymmetric 1,2-addition of arylboric acids to $\alpha$-diketones catalyzed by $\left[\left\{\mathrm{Rh}(\operatorname{coe})_{2} \mathrm{Cl}_{2}\right] / \mathbf{L 1 0}\right.$

\begin{tabular}{|c|c|c|c|c|}
\hline Entry & Substrate & $\mathrm{Ar}$ & Yield $^{b} / \%$ & $e e^{c} / \%$ \\
\hline 1 & $\mathrm{R}^{1}=\mathrm{R}^{2}=\mathrm{Ph}$ & 4- $\mathrm{MeC}_{6} \mathrm{H}_{4}$ & 97 & 97 \\
\hline 2 & $\mathrm{R}^{1}=\mathrm{R}^{2}=\mathrm{Ph}$ & $4-\mathrm{MeOC}_{6} \mathrm{H}_{4}$ & 99 & 97 \\
\hline 3 & $\mathrm{R}^{1}=\mathrm{R}^{2}=\mathrm{Ph}$ & $4-\mathrm{ClC}_{6} \mathrm{H}_{4}$ & 97 & 99 \\
\hline 4 & $\mathrm{R}^{1}=\mathrm{R}^{2}=\mathrm{Ph}$ & $4-\mathrm{FC}_{6} \mathrm{H}_{4}$ & 98 & 98 \\
\hline 5 & $\mathrm{R}^{1}=\mathrm{R}^{2}=\mathrm{Ph}$ & 4- $\mathrm{PhC}_{6} \mathrm{H}_{4}$ & 98 & 97 \\
\hline 6 & $\mathrm{R}^{1}=\mathrm{R}^{2}=\mathrm{Ph}$ & $3-\mathrm{MeOC}_{6} \mathrm{H}_{4}$ & 95 & 97 \\
\hline 7 & $\mathrm{R}^{1}=\mathrm{R}^{2}=\mathrm{Ph}$ & $4-\mathrm{MeC}_{6} \mathrm{H}_{4}$ & 54 & 95 \\
\hline 8 & $\mathrm{R}^{1}=\mathrm{R}^{2}=4-\mathrm{BrC}_{6} \mathrm{H}_{4}$ & 4- $\mathrm{MeC}_{6} \mathrm{H}_{4}$ & 93 & 98 \\
\hline 9 & $\mathrm{R}^{1}=\mathrm{R}^{2}=4-\mathrm{FC}_{6} \mathrm{H}_{4}$ & 4- $\mathrm{MeC}_{6} \mathrm{H}_{4}$ & 99 & 96 \\
\hline 10 & $\mathrm{R}^{1}=\mathrm{R}^{2}=4-\mathrm{MeOC}_{6} \mathrm{H}_{4}$ & 4- $\mathrm{MeC}_{6} \mathrm{H}_{4}$ & 98 & 97 \\
\hline 11 & $\mathrm{R}^{1}=\mathrm{R}^{2}=\mathrm{Me}$ & 4- $\mathrm{MeC}_{6} \mathrm{H}_{4}$ & 45 & 63 \\
\hline 12 & $\mathrm{R}^{1}=\mathrm{Me}, \mathrm{R}^{2}=\mathrm{Ph}$ & 4- $\mathrm{MeC}_{6} \mathrm{H}_{4}$ & 85 & 80 \\
\hline
\end{tabular}

${ }^{a}$ The reaction was carried out with $\alpha$-diketone $(0.25 \mathrm{mmol})$ and 1.5 equiv. of aryl boronic acid, in the presence of $3 \mathrm{~mol} \%$ [Rh], $3.3 \mathrm{~mol} \%$ ligand, and 0.1 $\mathrm{mol} / \mathrm{L}$ aq $\mathrm{KOH}(0.2 \mathrm{~mL}, 0.08$ equiv. $)$ in $1.0 \mathrm{~mL}$ of THF. ${ }^{b}$ Yield of isolated product. ${ }^{c}$ Determined by chiral HPLC.

同年, 杜海峰课题组 ${ }^{[40]}$ 也报道了手性烯烃-亚磺酸 胺配体 $\mathbf{L 1 1}$ 与 $\left[\mathrm{Rh}\left(\mathrm{C}_{2} \mathrm{H}_{4}\right)_{2} \mathrm{Cl}\right]_{2}$ 催化芳基硼酸与 1,2-二酮 的不对称加成(Eq. 12)，反应对于二芳基二酮均可获得 极好的反应结果，遗憾的是对于杂环如呋喃或噻吩二酮 反应只获得近乎消旋的加成产物(表 11).

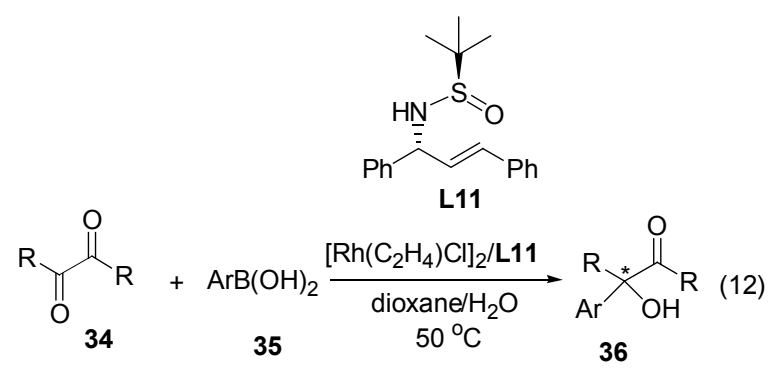

\section{5 不活泼酮}

与醛相比较, 不活泼酮的 1,2-加成困难的多, 尤其 是酮的芳基化反应更不容易．以往报道酮的芳基化反应 
表 $11 \mathrm{Rh}$-催化芳基嗍酸与 1,2-二酮的不对称加成 ${ }^{a}$

Table $11 \mathrm{Rh}(\mathrm{I})$-catalyzed asymmetric addition of arylboronic acids to $\alpha$-diketones

\begin{tabular}{|c|c|c|c|c|c|}
\hline Entry & $\mathrm{R}$ & $\mathrm{Ar}$ & Time/l & $\operatorname{eld}^{b} / \%$ & $e e^{c} / \%$ \\
\hline 1 & $\mathrm{Ph}$ & $4-\mathrm{MeOC}_{6} \mathrm{H}_{4}$ & 3 & 99 & 98 \\
\hline 2 & $\mathrm{Ph}$ & 4- $\mathrm{MeC}_{6} \mathrm{H}_{4}$ & 9 & 88 & 98 \\
\hline 3 & $\mathrm{Ph}$ & $4-t-\mathrm{BuC}_{6} \mathrm{H}_{4}$ & 4 & 79 & 98 \\
\hline 4 & $\mathrm{Ph}$ & $4-\mathrm{FC}_{6} \mathrm{H}_{4}$ & 7 & 56 & 99 \\
\hline 5 & $\mathrm{Ph}$ & 4- $\mathrm{AcC}_{6} \mathrm{H}_{4}$ & 8 & 61 & 99 \\
\hline 6 & $\mathrm{Ph}$ & $3-\mathrm{ClC}_{6} \mathrm{H}_{4}$ & 4 & 99 & 99 \\
\hline 7 & $\mathrm{Ph}$ & $2,4-\mathrm{CH}_{3} \mathrm{C}_{6} \mathrm{H}_{3}$ & 12 & 99 & 97 \\
\hline 8 & $\mathrm{Ph}$ & Naphthyl & 12 & 99 & 98 \\
\hline 9 & $\mathrm{Ph}$ & Naphthyl & 6 & 76 & 97 \\
\hline 10 & $\mathrm{Ph}$ & $2-\mathrm{MeC}_{6} \mathrm{H}_{4}$ & 9 & 57 & 96 \\
\hline 11 & $\mathrm{Ph}$ & Styryl & 11 & 40 & 88 \\
\hline 12 & 4- $\mathrm{MeC}_{6} \mathrm{H}_{4}$ & $4-\mathrm{BrC}_{6} \mathrm{H}_{4}$ & 4 & 87 & 97 \\
\hline 13 & $4-\mathrm{MeOC}_{6} \mathrm{H}_{4}$ & $3-\mathrm{MeOC}_{6} \mathrm{H}_{4}$ & 12 & 99 & 99 \\
\hline 14 & $4-\mathrm{MeOC}_{6} \mathrm{H}_{4}$ & Furyl & 11 & 99 & 4 \\
\hline 15 & $4-\mathrm{MeOC}_{6} \mathrm{H}_{4}$ & Thienyl & 11 & 98 & 16 \\
\hline
\end{tabular}

${ }^{a}$ Reactions were carried out in $2.0 \mathrm{mmol}$ of scale and $3.0 \mathrm{~mL}$ of solvent for 0.1 mol\% catalyst loading and in $0.4 \mathrm{mmol}$ scale and $1.5 \mathrm{~mL}$ of solvent for 1.0 and $3.0 \mathrm{~mol} \%$ catalyst loading at $50{ }^{\circ} \mathrm{C} .{ }^{b}$ Isolated yield. ${ }^{c}$ The $e e$ was determined by chiral HPLC.

主要是使用二芳基锌试剂, 然而考虑到反应试剂的制备 与稳定性, 芳基硼酸则显得更有应用价值. 因此, 如何 实现芳基硼酸与酮的 1,2-加成具有非常重要的理论与现 实意义. 2006 年, $\mathrm{Lu}$ 等 ${ }^{[41]}$ 利用手性正离子钯化合物 37 (图9) 实现了分子内芳基嗍酸与酮的加成反应获得了理 想的反应结果. 该部分研究工作在 2009 年 Wiley 出版的 《Modern Arylation Methods》一书中已有提及, 在此不再 赘述 ${ }^{[19]}$.

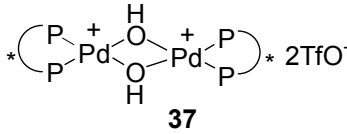

$$
\begin{aligned}
& { }^{*} C_{\mathrm{P}}^{\mathrm{P}}=(R) \text {-BINAP }
\end{aligned}
$$

图 9 手性正离子钯化合物 $\mathbf{3 7}$

Figure 9 chiral positive ions palladium compound $\mathbf{3 7}$

此外, Sarpong 等 ${ }^{[42]}$ 也报道了 $[\mathrm{Rh}(\operatorname{cod}) \mathrm{OH}]_{2}$ 与手性 二茂铁双膦配体催化分子内芳基嗍烷对酮不对称加成 反应(Eq. 13), 反应的对映选择性均在 $92 \%$ 以上(表 12), 不足的是反应所需的催化剂及配体的量较大.

最近, Lam 等 ${ }^{[43]}$ 报道了使用 $\mathrm{Rh}$ 与手性亚酰胺一烯化 合物催化 $N$-杂酮的分子内芳基化反应(Eq. 14), 反应可 获得高收率及对映选择性的 $\mathrm{N}$-杂环加成产物 (Up to 95\% yield, 91\% ee).

与分子内的加成反应相比较, 分子间芳基硼酸与酮 的不对称加成则难的多. 虽然 Itami ${ }^{[44]}$ 与 Sakai 等 ${ }^{[45]}$ 分别 报道了零价 $\mathrm{Ni}$ 和 $[\mathrm{Rh}(\mathrm{COD}) \mathrm{OH}]_{2}$ 与负电子的双膦配体催 化芳基嗍酸与苯乙酮的加成反应可获得极好的收

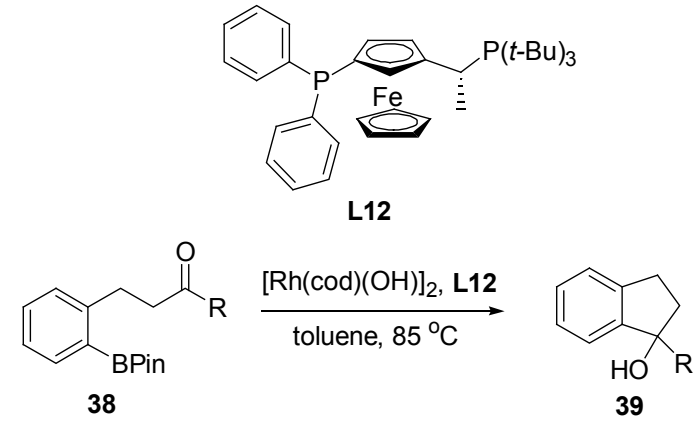

表 12 不对称羟芳基化反应 ${ }^{a}$

Table 12 Enantioselective hydroarylation reactions

\begin{tabular}{clcc}
\hline Entry & \multicolumn{1}{c}{$\mathrm{R}$} & $\mathrm{Yield}^{b} / \%$ & $e e^{c} / \%$ \\
\hline 1 & $\mathrm{Ph}$ & 78 & 94 \\
2 & $3-\mathrm{MeOC}_{6} \mathrm{H}_{4}$ & 85 & 95 \\
3 & 4- $\mathrm{MeC}_{6} \mathrm{H}_{4}$ & 90 & 94 \\
4 & 2-Naphthyl & 50 & 93 \\
5 & 4-FC $\mathrm{H}_{4}$ & 54 & 92 \\
6 & $\mathrm{Me}$ & 90 & 95 \\
\hline
\end{tabular}

${ }^{a}$ Reaction conditions: $5.0 \mathrm{~mol} \%[\mathrm{Rh}(\mathrm{cod})(\mathrm{OH})]_{2}, 11 \mathrm{~mol} \% \mathrm{L11}, 0.1 \mathrm{~mol} / \mathrm{L}$ in PhMe. ${ }^{b}$ Isolated yield. ${ }^{c}$ Determined by chiral HPLC.

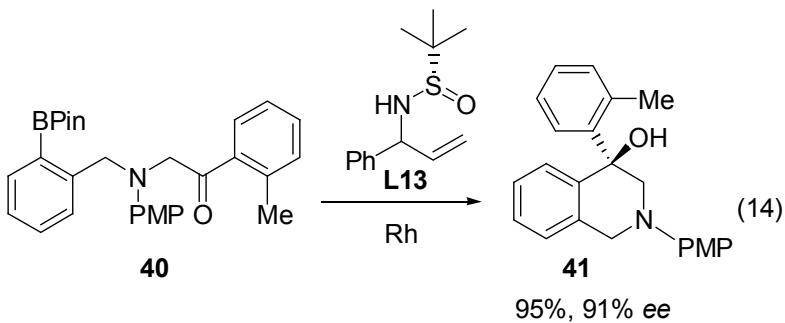

率，但均只获得消旋的加成产物，金属催化的分子间芳 基硼酸与不活泼酮的不对称 1,2-加成一直没有报道. 直 至最近 $\mathrm{Hu}$ 等 ${ }^{[46]}$ 第一次报道了使用手性双烯配体与 $\mathrm{Rh}$ 不对称催化芳基硼酸与苯乙酮的 1,2-加成反应(Eq. 15), 不仅获得很好的收率，反应也获得了中等的对映选择性 (表 13). 虽然反应的对映选择性不理想，但该方法的报 道为以后的研究提供了非常重要的理论指导意义.
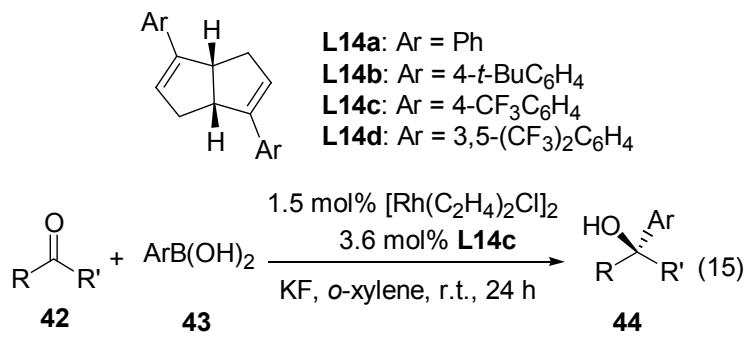

此外, 最近我们课题组 ${ }^{[29]}$ 发现使用手性双膦配体 L7 与 $\left[\mathrm{RhCl}\left(\mathrm{C}_{2} \mathrm{H}_{4}\right)_{2}\right]_{2}$ 也可催化芳基硼酸与不活泼酮如苯 乙酮的不对称加成反应(Eq. 16), 反应可获得极好的对 应选择性 $(96 \% e e)$, 遗憾的是反应的活性不高(产率 
表 $13 \mathrm{Rh}(\mathrm{I}) / \mathbf{1 4 c}$ 催化芳基硼酸与不活泼酮的不对称加成反 应 $^{a}$

Table $13 \mathrm{Rh}(\mathrm{I}) / \mathbf{1 4 c}$-catalyzed addition reactions of arylboronic acid with unactive ketones

\begin{tabular}{cllcc}
\hline Entry & \multicolumn{1}{c}{ Ketone } & \multicolumn{1}{c}{$\mathrm{Ar}$} & Yield $^{b} / \%$ & $e e^{c} / \%$ \\
\hline 1 & Propiophenone & $4-\mathrm{MeC}_{6} \mathrm{H}_{4}$ & 83 & 47 \\
2 & Propiophenone & $3-\mathrm{MeC}_{6} \mathrm{H}_{4}$ & 85 & 39 \\
3 & $\alpha$-Tetralone & $\mathrm{Ph}$ & 81 & 36 \\
4 & $\alpha$-Tetralone & $4-\mathrm{MeC}_{6} \mathrm{H}_{4}$ & 80 & $68^{d}$ \\
5 & $\alpha$-Tetralone & $3-\mathrm{MeC}_{6} \mathrm{H}_{4}$ & 84 & 43 \\
6 & $\alpha$-Tetralone & $4-\mathrm{MeOC}_{6} \mathrm{H}_{4}$ & 84 & $49^{e}$ \\
7 & 1 -Indanone & $4-\mathrm{MeC}_{6} \mathrm{H}_{4}$ & 83 & 56 \\
\hline
\end{tabular}

${ }^{a}$ Reaction conditions: ketone $(0.25 \mathrm{mmol})$, arylboronic acid (2 equiv.), KF (3 equiv.), $o$-xylene $(1.0 \mathrm{~mL}),\left[\mathrm{Rh}\left(\mathrm{C}_{2} \mathrm{H}_{4}\right)_{2} \mathrm{Cl}\right]_{2}(1.5 \mathrm{~mol} \%)$. L14c $(3.6 \mathrm{~mol} \%)$. ${ }^{b}$ Isolated yield. ${ }^{c}$ Determined by HPLC. ${ }^{d} 5.0 \mathrm{~mol} \% \mathrm{Rh}(\mathrm{I}) / 6 \mathrm{~mol} \% \mathrm{L14c}$ at 0 ${ }^{\circ} \mathrm{C}$ for $7 \mathrm{~d} .{ }^{e}$ Reaction at $60{ }^{\circ} \mathrm{C}$.<smiles>CC(=O)c1ccccc1</smiles>

45

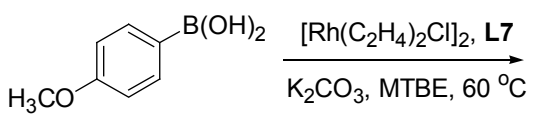

46
$25 \%$ yield, $96 \%$ ee<smiles>COc1ccc([C@@](C)(O)c2ccccc2)cc1</smiles>

$25 \%$ ). 作者进一步研究发现使用缼电子膦配体可大大 提高反应的活性, 进一步的研究工作正在进行.

\section{6 总结与展望}

到目前为止, 金属催化的芳基硼酸与酮的加成反应 虽然取得了一定的进展, 但以往报道较为成功的主要是 芳基硼酸与酮如静红、三氟甲基酮、 $\alpha$-酮酯及 1,2 -二酮 类化合物或者分子内酮的不对称加成. 不活泼酮如苯乙 酮的 1,2-加成反应目前鲜有报道且结果也不理想. 目前 Sakai 与 $\mathrm{Hu}$ 研究小组报道使用缺电子的双膦配体或者 双烯配体可很好的解决一般酮的低反应活性问题, 尤其 是 Sakai 报道的使用负电子双膦配体通过与金属络合后 再与底物络合后可非常有效的活化反应底物从而可获 得高收率的 1,2-加成产物. 虽然上述两例报道反应的对 映选择性不尽如人意, 但为随后的金属催化芳基硼酸与 不活泼酮的不对称 1,2-加成提供了很有效的理论指导, 尤其是对手性配体的设计合成提供了较为明确的设计 思路. 最近作者研究发现使用具有手性膦中心的双膦配 体不仅可以很好的催化芳基嗍酸与三氟甲基苯乙酮获 得高收率及高对映选择性的加成产物, 也可催化分子间 芳基嗍酸与不活泼酮如苯乙酮获得高对映选择性的加 成产物, 遗憾的是反应的活性不高. 根据 Sakai 研究小 组报道的研究结果, 作者认为设计并合成强负电子的具
有手性膦中心的双膦配体可解决分子间芳基硼酸与不 活泼酮加成反应中存在的低活性及低对映选择性问题. 目前该方面的研究也正在进行并取得了可喜的进展，作 者相信在不久的将来对于目前分子间芳基硼酸与不活 泼酮所存在的问题应可很好的解决并应用到更多的有 机合成及药物合成中.

\section{References}

[1] For reviews, see: (a) Jacobsen, E. N.; Pfaltz, A.; Yamamoto, H. Comprehensive Asymmetric Catalysis, 1 st ed., Springer, Berlin, 1999.

(b) Pu, L.; Yu, H.-B. Chem. Rev. 2001, 101, 757.

(c) Fagnou, K.; Lautens, M. Chem. Rev. 2003, 103, 169.

(d) Denmark, S. E.; Fu, J. Chem. Rev. 2003, 103, 2763.

(e) Garcia, C.; Martin, V. S. Curr. Org. Chem. 2006, 10, 1849.

(f) Riant, O.; Hannedouche, J. Org. Biomol. Chem. 2007, 5, 873.

(g) Tian, P.; Dong, H.-Q.; Lin, G.-Q. ACS Catal. 2011, 95.

[2] (a) Romero, D. L. Ann. Rep. Med. Chem. 1994, 29, 123.

(b) de Clercq, E. Rev. Med. Virol. 2009, 19, 287.

(c) Wan, Z.-K.; Chenail, E.; Li, H.-Q.; Kendall, C.; Wang, Y.; Gingras, S.; Xiang, J.; Massefski, W. W.; Mansour, T. S.; Saiah, E. J. Org. Chem. 2011, 76, 7048.

(d) Julian, L.; Wang, D. Z.; Bostick, T.; Caille, S.; Choi, R.; DeGraffenreid, M.; Di, Y.; He, X.; Hungate, R. W.; Jaen, J. C.; Liu, J.; Monshouwer, M.; McMinn, D.; Rew, Y.; Sudom, A.; Sun, D.; Tu, H.; Ursu, S.; Walker, N.; Yan, X.; Ye, Q.; Powers, J. P. J. Med. Chem. 2008, 51, 3953.

(e) Kopecky, D. J.; Jiao, X. Y.; Fisher, B.; McKendry, S.; Labelle, M.; Piper, D. E.; Coward, P.; Shiau, A. K.; Escaron, P.; Danao, J.; Chai, A.; Jaen, J.; Kayser, F. Bioorg. Med. Chem. Lett. 2012, 22, 2407.

[3] (a) Marshall, R. L.; Muderawan, I. W.; Young, D. J. J. Chem. Soc., Perkin Trans. 2 2000, 957.

(b) Li, C.-J.; Meng, Y. J. Am. Chem. Soc. 2000, 122, 9538.

[4] (a) Dosa, P. I.; Ruble, J. C.; Fu, G. C. J. Org. Chem. 1997, 62, 444. (b) Huang, W.-S.; Hu, Q.-S.; Pu, L. J. Org. Chem. 1999, 64, 7940. (c) Bolm, C.; Hermanns, N.; Hildebrand, J. P.; Muniz, K. Angew. Chem., Int. Ed. 2000, 39, 3465.

(d) Bolm, C.; Rudolph, J. J. Am. Chem. Soc. 2002, 124, 14850. (e) Montserrat, F.; Xavier, V.; Lluis, S.; Miquel, A. P.; Antoni, R. J. Org. Chem. 2004, 69, 2532.

(f) Braga, A. L.; Lüdtke, D. S.; Vargas, F.; Paixão, M. W. A. Chem. Commun. 2005, 19, 2512.

[5] Tomita, D.; Wada, R.; Kanai, M.; Shibasaki, M. J. Am. Chem. Soc. $\mathbf{2 0 0 5}, 127,4138$.

[6] Weber, B.; Seebach, D. Tetrahedron 1994, 50, 7473.

[7] Sakai, M.; Ueda, M.; Miyaura, N. Angew. Chem., Int. Ed. 1998, 37, 3279 .

[8] Using rhodium catalysis: (a) Sakai, M.; Ueda, M.; Miyaura, N. Angew. Chem., Int. Ed. 1998, 37, 3279.

(b) Focken, T.; Rudolph, J.; Bolm, C. Synthesis 2005, 429.

(c) Suzuki, K.; Ishii, S.; Kondo, K.; Aoyama, T. Synlett 2006, 648.

(d) Suzuki, K.; Kondo, K.; Aoyama, T. Synthesis 2006, 1360.

(e) Arao, T.; Suzuki, K.; Kondo, K.; Aoyama, T. Synthesis 2006, 3809 .

(f) Duan, H. F.; Xie, J. H.; Shi, W. J.; Zhang, Q.; Zhou, Q. L. Org. Lett. 2006, 8, 1479.

(g) Jagt, R. B. C.; Toullec, P. Y.; de Vries, J. G.; Feringa, B. L.; Minnaard, A. J. Org. Biomol. Chem. 2006, 4, 773. 
(h) No€el, T.; Vandyck, K.; Van der Eycken, J. Tetrahedron 2007, $63,12961$.

(i) Nishimura, T.; Kumamoto, H.; Nagaosa, M.; Hayashi, T. Chem. Commun. 2009, 5713.

(j) Clarke, E. F.; Rafter, E.; M€uller-Bunz, H.; Higham, L. J.; Gilheany, D. G. J. Organomet. Chem. 2011, 696, 3608.

[9] Using cobalt catalysis: Karthikeyan, J.; Jeganmohan, M.; Cheng, C.-H. Chem. Eur. J. 2010, 16, 8989.

[10] Using nickel catalysis: (a) Arao, T.; Kondo, K.; Aoyama, T. Tetrahedron Lett. 2007, 48, 4115.

(b) Yamamoto, K.; Tsurumi, K.; Sakurai, F.; Kondo, K.; Aoyama, T. Synthesis 2008, 3585.

(c) Sakupai, F.; Kondo, K.; Aoyama, T. Chem. Pharm. Bull. 2009, $57,511$.

(d) Sakurai, F.; Kondo, K.; Aoyama, T. Tetrahedron Lett. 2009, 50, 6001.

[11] Using copper catalysis: Tomita, D.; Kanai, M.; Shibasaki, M. Chem. Asian J. 2006, 1, 161.

[12] Using ruthenium catalysis: (a) Yamamoto, Y.; Kurihara, K.; Miyaura, N. Angew. Chem., Int. Ed. 2009, 48, 4414.

(b)Yamamoto, Y.; Shirai, T.; Watanabe, M.; Kurihara, K.; Miyaura, N. Molecules 2011, 16, 5020.

(c) Yamamoto, Y.; Shirai, T.; Miyaura, N. Chem. Commun. 2012, $48,2803$.

(d) Li, K.; Hu, N.-F.; Luo, R.-S.; Yuan, W.-C.; Tang, W. J. Org. Chem. 2013, 78, 6350.

[13] Zhang, R.; Xu, Q.; Zhang, X.; Zhang, T.; Shi, M. Tetrahedron: Asymmetry 2010, 21, 1928.

[14] Luo, F.; Pan, C. D.; Cheng, J. Chin. J. Org. Chem. 2010, 30, 633 (in Chinese).

(罗芳, 潘长多, 成江, 有机化学, 2010, 30, 633.)

[15] (a) Shintani, R.; Inoue, M.; Hayashi, T. Angew. Chem., Int. Ed. 2006, 45, 3353.

(b) Toullec, P. Y.; Jagt, R. B. C.; de Vries, J. G.; Feringa, B. L.; Minnaard, A. J. Org. Lett. 2006, 8, 2715.

(c) Shintani, R.; Takatsu, K.; Hayashi, T. Chem. Commun. 2010, 46, 6822 .

(d) Martina, S. L. X.; Jagt, R. B. C.; de Vries, J. G.; Feringa, B. L.; Minnaard, A. J. Chem. Commun. 2006, 4093.

(e) Duan, H. F.; Xie, J. H.; Qiao, X. C.; Wang, L. X.; Zhou, Q. L. Angew. Chem., Int. Ed. 2008, 47, 4351.

(f) Cai, F.; Pu, X.; Qi, X.; Lynch, V.; Radha, A.; Ready, J. M. J. Am. Chem. Soc. 2011, 133, 18066.

(g) Zhu, T.-S.; Jin, S.-S.; Xu, M.-H. Angew. Chem., Int. Ed. 2012, 51, 780 .

(h) Feng, X.; Nie, Y.; Yang, J.; Du, H. Org. Lett. 2012, 14, 624.

[16] Toullec, P. Y.; Jagt, R. B. C.; Vries, J. G.; Feringa, B. L.; Minnaard, A. J. Org. Lett. 2006, 8, 2715.

[17] Jagt, R. B. C.; Toullec, P. Y.; de Vries, J. G.; Feringa, B. L.; Minnaard, A. J. Org. Biomol. Chem. 2006, 4, 773.

[18] Shintani, R.; Inoue, M.; Hayashi, T. Angew. Chem., Int. Ed. 2006, 45, 3353.

[19] Ackermann, L. Modern Arylation Methods, Wiley-VCH, Weinheim, Germany, 2009, pp. 271 310.

[20] Lai, H. S.; Huang, Z. Y.; Wu, Q.; Qin, Y. J. Org. Chem. 2009, 74, 283.

[21] Gui, J. Y.; Chen, G. H.; Cao, P.; Liao, J. Tetrahedron: Asymmetry 2012, 23, 554.

[22] (a) Yamamoto, Y.; Kurihara, K.; Miyaura, N. Angew. Chem., Int. Ed. 2009, 48, 4414.

(b) Yamamoto, Y.; Shirai, T.; Watanabe, M.; Kurihara, K.; Miyaura, N. Molecules 2011, 16, 5020. (c) Yamamoto, Y.; Shirai, T.; Miyaura, N. Chem. Commun. 2012 48, 2803.

[23] Yamamoto, Y.; Yohd, M.; Shirai, T.; Ito, H.; Miyaura, N. Chem. Asian J. 2012, 7, 2246.

[24] (a) Kaur, H.; Zinn, F. K.; Stevens, E. D.; Nolan, S. P. Organometallics 2004, 23, 1157.

(b) Diez-Gonzalez, S.; Kaur, H.; Zinn, F. K.; Stevens, E. D.; Nolan, S. P. J. Org. Chem. 2005, 70, 4784.

(c) Lebel, H.; Davi, M.; Diez-Gonzalez, S.; Nolan, S. P. J. Org. Chem. 2007, 72, 144.

(d) Laitar, D. S.; Tsui, E. Y.; Sadighi, J. P. J. Am. Chem. Soc. 2006, 128, 11036 .

(e) Jurkauskas, V.; Sadighi, J. P.; Buchwald, S. L. Org. Lett. 2003, $5,2417$.

(f) Laitar, D. S.; Müller, P.; Sadighi, J. P. J. Am. Chem. Soc. 2005, 127, 17196.

[25] Shintani, R.; Takatsu, K.; Hayashi, T. Chem. Commun. 2010, 46, 6822.

[26] Liu, Z.; Gu, P.; Shi, M.; McDowell, P.; Li, G. G. Org. Lett. 2011, 13, 2314.

[27] Martina, S. L. X.; Jagt, R. B. C.; Vries, J. G.; Feringa B. L.; Minnaard, A. J. Chem. Commun. 2006, 4093.

[28] Jumde, V. R.; Facchetti, S.; Iuliano, A. Tetrahedron: Asymmetry 2010, 21, 2775.

[29] Luo, R.-S.; Li, K.; Hu, Y. L.; Tang, W. J. Adv. Synth. Catal. 2013, $355,1297$.

[30] (a) Kiesewetter, D. O.; Silverton, J. V.; Eckelman, W. C. J. Med. Chem. 1995, 38, 1711.

(b) Kiesewetter, D. O.; Carson, R. E.; Jagoda, E. M.; Endres, C. J.; Der, M. G.; Herscovitch, P.; Eckelman, W. C. Bioorg. Med. Chem. $1997,5,1555$.

(c) Skaddan, M. B.; Kilbourn, M. R.; Snyder, S. E.; Sherman, P. S.; Desmond, T. J.; Frey, K. A. J. Med. Chem. 2000, 43, 4552.

(d) Selent, J.; Brandt, W.; Pamperin, D. Goeber, B. Bioorg. Med. Chem. 2006, 14, 1729.

[31] (a) He, P.; Lu, Y.; Hu, Q.-S. Tetrahedron Lett. 2007, 48, 5283. (b) He, P.; Lu, Y.; Dong, C.-G.; Hu, Q.-S. Org. Lett. 2007, 9, 343.

[32] Xie, J.-H.; Zhu, S.-F.; Zhou, Q.-L. Chem. Soc. Rev. 2012, 41, 4126.

[33] Duan, H.-F.; Xie, J.-H. Qiao, X.-C. Wang, L.-X.; Zhou, Q.-L. Angew. Chem., Int. Ed. 2008, 47, 4351.

[34] Cai, F.; Pu, X. T.; Qi, X. B.; Lynch, V.; Radha, A.; Ready, J. M. J. Am. Chem. Soc. 2011, 133, 18066.

[35] (a) Jin, S.-S.; Wang, H.; Xu, M.-H. Chem. Commun. 2011, 47, 7230 .

(b) Qi, W.-Y.; Zhu, T.-S.; Xu, M.-H. Org. Lett. 2011, 13, 3410.

(c) Thaler, T.; Guo, L.-N.; Steib, A. K.; Raducan, M.; Karaghiosoff, K.; Mayer, P.; Knochel, P. Org. Lett. 2011, 13, 3182.

(d) Feng, X. Wang, Y.; Wei, B.; Yang, J.; Du, H. Org. Lett. 2011, 13,3300 .

(e) Chen, G.; Gui, J.; Li, L.; Liao, J. Angew. Chem., Int. Ed. 2011, 50,7681 .

(f) Feng, X.; Wei, B.; Yang, J.; Du, H. Org. Biomol. Chem. 2011, 9, 5927.

(g) Xue, F.; Li, X.; Wan, B. J. Org. Chem. 2011, 76, 7256.

(h) Wang, Y.; Feng, X.; Du, H. Org. Lett. 2011, 13, 4954.

[36] Zhu, T.-S.; Jin, S.-S.; Xu, M.-H. Angew. Chem., Int. Ed. 2012, 51, 780.

[37] Jung, J.-K.; Johnson, B. R.; Duong, T.; Decaire, M.; Uy, J.; Gharbaoui, T.; Boatman, P. D.; Sage, C. R.; Chen, R.; Richman, J. G.; Connolly, D. T.; Semple, G. J. Med. Chem. 2007, 50, 1445.

[38] Singh, S. B.; Zink, D. L.; Quamina, D. S.; Pelaez, F.; Teran, A.; Felock, P.; Hazuda, D. J. Tetrahedron Lett. 2002, 43, 2351. 
[39] Ganci, G. R.; Chisholm, J. D. Tetrahedron Lett. 2007, 48, 8266.

[40] Feng, X. Q.; Nie, Y. Z.; Yang, J.; Du, H. F. Org. Lett. 2012, 14, 624.

[41] Liu, G. X.; Lu, X. Y. J. Am. Chem. Soc. 2006, 128, 16504.

[42] Gallego, G. M.; Sarpong, R. Chem. Sci. 2012, 3, 1338.

[43] Low, D. W.; Pattison, G.; Wieczysty, M. D.; Churchill, G. H.; Lam,
H. W. Org. Lett. 2012, 14, 2548.

[44] Bouffard, J.; Itami, K. Org. Lett. 2009, 11, 4410.

[45] Korenaga, T.; Ko, A.; Uotani, K.; Tanaka, Y.; Sakai, T. Angew. Chem., Int. Ed. 2011, 50, 10703.

[46] Liao, Y.-X.; Xing, C.-H.; Hu, Q.-S. Org. Lett. 2012, 14, 1544. 